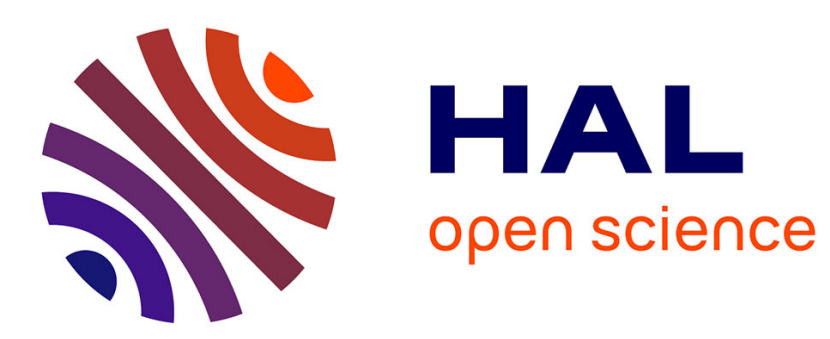

\title{
The three-dimensional seismological model a priori constrained: Confrontation with seismic data
}

\author{
Y. Ricard, Henri-Claude Nataf, J.-P. Montagner
}

\section{To cite this version:}

Y. Ricard, Henri-Claude Nataf, J.-P. Montagner. The three-dimensional seismological model a priori constrained: Confrontation with seismic data. Journal of Geophysical Research: Solid Earth, 1996, 101 (4), pp.8457-8472. 10.1029/95JB03744 . hal-02046757

\section{HAL Id: hal-02046757 \\ https://hal.science/hal-02046757}

Submitted on 7 Dec 2020

HAL is a multi-disciplinary open access archive for the deposit and dissemination of scientific research documents, whether they are published or not. The documents may come from teaching and research institutions in France or abroad, or from public or private research centers.
L'archive ouverte pluridisciplinaire HAL, est destinée au dépôt et à la diffusion de documents scientifiques de niveau recherche, publiés ou non, émanant des établissements d'enseignement et de recherche français ou étrangers, des laboratoires publics ou privés. 


\title{
The three-dimensional seismological model a priori constrained: Confrontation with seismic data
}

\author{
Yanick Ricard \\ Ecole Normale Supérieure de Lyon, Lyon, France \\ Henri-Claude Nataf \\ Ecole Normale Supérieure de Paris, Paris, France \\ Jean-Paul Montagner \\ Institut de Physique du Globe, Paris, France
}

\begin{abstract}
We compare the predictions of an a priori model of the upper mantle with seismic observations of surface waves and eigenmodes. The 3-Dimensional Seismological Model A Priori Constrained (3SMAC) has been developed by Nataf and Ricard [1996]. It is based on the interpretation by geodynamicists of the near surface layers of the Earth; on distributions of temperature, pressure, and composition as a function of depth; and then on estimates of seismic parameters (density, velocities, attenuations) from solid state laboratory measurements as a function of temperature and pressure. The 3SMAC predictions are confronted with observations consisting of phase velocities for Love and Rayleigh waves in the period range of 70-250 s [Montagner and Tanimoto, 1990]. We first show that tomographic inversions applied to 3SMAC synthetics induce a strong smoothing of the heterogeneities. This casts doubt on the meaning of the spectra of mantle heterogeneities revealed by tomography. We then show that most of the Love and Rayleigh fundamental mode observations for periods less than $200 \mathrm{~s}$ are satisfactorily predicted by 3SMAC. The major differences come from the seismic velocities under the Red Sea and Southeast China, which are much slower than what is estimated from 3SMAC, as well as those under Greenland, which are not as fast as the other cratonic areas. Because the lithosphere is thinner than $100 \mathrm{~km}$ under oceans and thinner than $300 \mathrm{~km}$ under continents in 3SMAC, we suggest that the existence of deeper lithospheric anomalies as proposed in many tomographic models is mostly due to a spurious effect of the inversion rather than implied by surface wave data. Half of the variance of the degree 2 anomaly mapped by low-degree eigenmode observations can be explained by lithospheric velocity structures. The other half is highly correlated with the distribution of deep slabs, but its amplitude is a factor of 3 or 4 larger than that predicted by 3SMAC. The lithospheric anomalies present a degree 6 pattern well correlated with the distribution of hotspots even when the thermal anomalies that could be associated with plumes are not included in 3SMAC. Our results emphasize the importance of giving very close attention to "surface corrections" in tomographic models.
\end{abstract}

\section{Introduction}

The 3-Dimensional Seismological Model A Priori Constrained (3SMAC) model discussed by Nataf and Ricard [1996] can be used to perform various experiments on seismic wave propagation. First, it can be used to

Copyright 1996 by the American Geophysical Union.

Paper number 95JB03744.

0148-0227/96/95JB-03744\$05.00 perform synthetic experiments in order to quantify the ability of tomographic methods in recovering the Earth structure. This will give us an estimate of the degradation (aliasing, smoothing, blurring, and so on) associated with mantle imaging. Second, it can be compared with real seismic data and therefore tell us what part of the global seismic observations are likely related to lithospheric features. Of course the two points are closely linked, as it would not be useful to compute synthetics unless 3SMAC gave a realistic picture of the major lithospheric anomalies. Our investigations into the resolution of seismic tomography and into the effects of 
lithospheric heterogeneities are somewhat akin to what has been done by others [e.g., Kawakatsu, 1983; Snieder et al., 1991; Pulliam and Johnson; 1992, Mochizuki, 1993; Trampert and Woodhouse, 1995]. The main differences are that $3 \mathrm{SMAC}$ is much more complex than the models on which resolution tests have been performed and that 3SMAC proposes a mantle structure built independently of previous seismic studies.

A direct comparison between 3SMAC and a threedimensional tomographic model deduced from observations could be easily done. However, even though 3SMAC and any present-day tomographic model have slow ridges and fast cratonic areas in common, there is clearly no quantitative agreement between them. As an example, there are no velocity anomalies under oceans in $3 \mathrm{SMAC}$ at depth greater than $95 \mathrm{~km}$ except under localized hotspots. Such a confinement of the anomalies in the shallowest layers cannot be found in tomographic models, as it would demand a vertical resolution amounting to a fraction of the lithospheric thickness. Up to now the vertical resolution of large-scale tomographic models does not seem to be smaller than $100 \mathrm{~km}$ even above areas where the data density is the highest [Grand, 1994].

Nataf and Ricard [1996] have already shown that 3SMAC predicts $\mathrm{P}$ and $\mathrm{S}$ travel time anomaly amplitudes, dependences of SS-S and PP-P under ocean floors, and SS-S versus PP-P residuals in rough agreement with the observations compiled by Woodward and Masters [1991]. In the present paper we will compare 3SMAC predictions with observations of Love and Rayleigh waves that are sampling the upper part of the upper mantle and with observations of low-degree eigenmodes sensitive to deeper velocity anomalies such as those located in the transition zone.

Many tomographic models built from surface wave velocity observations are formally or implicitly obtained after a two-stage inversion: The first converts a discrete data set of phase velocity observations for various earthquake/recorder couples into continuous geographic distributions of phase velocity at different periods, and the second takes advantage of the variable depth sensitivity of surface waves at different periods to invert for the depth dependence of the seismological parameters. As we want to stay as close as possible to the observations, we do not compare $3 \mathrm{SMAC}$ with any tomographic model. Instead we directly compare the maps of phase velocities that can be obtained from observations on a given ray coverage to the equivalent maps obtained by using 3SMAC synthetics computed on the same ray paths. In the future, a complete depth dependent inversion of 3 SMAC synthetics could be done to test the accuracy of the algorithms, using the dispersion of surface waves to recover the radial velocity distribution.

For a given period, Love waves are more sensitive to the near-surface structure than Rayleigh waves, and both types of waves are more sensitive to shallow structure at short periods than at long periods. Thus one guesses that 3SMAC should be a better seismological model at $70 \mathrm{~s}$ than at $250 \mathrm{~s}$ and a better model for
Love than for Rayleigh waves if the regionalization and parameters used in building 3SMAC have been properly chosen. The differences between 3SMAC predictions and observations should increase at larger periods where the effect of deep-seated heterogeneities should dominate the signal.

\section{SMAC Surface Wave Velocities}

In this paper we derive the phase slowness anomaly under the geometric optics approximation. The local phase velocity $C(T, \theta, \phi)$ for a period $T$ is simply computed from the seismic properties of the vertical column located below. The observed phase slowness $1 / C_{\Delta}(T)$ is the integral of local phase slownesses along the source receiver great circle path $\Delta$ [Sato and Santo, 1969; Jordan, 1978],

$$
\frac{1}{C_{\Delta}(T)}=\frac{1}{\Delta} \int_{\Delta} \frac{1}{C(T, \theta, \phi)} d s .
$$

This approximation has been widely used in global upper mantle tomography [e.g., Woodhouse and Dziewonskı, 1984; Nakanishı and Anderson, 1984a, b; Nataf et al., 1986; Montagner and Tanımoto, 1990]. It is a good approximation if the wavelength of the seismic signal is small compared to the spatial wavelength of heterogeneities. Of course, for the real Earth the slowness is also affected by heterogeneities lying off the path, and the path itself, owing to refraction, does not exactly follow a great circle. More sophisticated methods use the seismic wave polarizations or amplitudes to go beyond the great circle approximation [Woodhouse and Wong, 1986; Laske et al., 1994].

We compare the 3SMAC predictions with phase velocity observations made by Montagner and Tanimoto [1990] (hereafter referred to as M\&T) at 11 periods ranging from $250 \mathrm{~s}$ to $70 \mathrm{~s}$. These periods closely correspond to those of the 22 following eigenmodes 0T31, 0T40, 0T49, 0T58, 0T67, 0T77, 0Т86, 0T95, 0T104, 0T113, 0T122, 0S32, 0S43, 0S54, 0S65, 0S76, 0S87, 0S98, 0S108, 0S119, 0S129, and 0S140. To perform the comparisons between observations and synthetics, we identify the phase velocity at a given period with the phase velocity of the eigenmode having the closest period.

M\&T observations make use of 2184 Love and 3610 Rayleigh wave paths. These paths follow either the shortest arc at the surface of the Earth (G1 and R1 paths) or the longest (G2 and R2 paths). The data set comes from 107 earthquakes recorded by the GEOSCOPE [Romanowıcz et al., 1984] and the Global Digital Seismograph Network (GDSN) [Peterson and Hutt, 1982]. The data set was carefully chosen in order to obtain a rather homogeneous coverage of the Earth surface.

To compute synthetics of phase velocity anomalies with 3SMAC, we must first compute the local slowness anomalies, which are then integrated along paths. As $3 \mathrm{SMAC}$ is defined on a $2^{\circ}$ by $2^{\circ}$ grid, we build the 16,200 
columns of seismic parameters (seismic velocities, density, attenuation). Then we compute the periods of the 22 eigenmodes under consideration, using the program MINOS written by J. H. Woodhouse, F. Gilbert, and G. Masters. The correspondence between the phase velocity $C_{l}$ and the period $T_{l}$ of the mode of degree $l$ is given by the following equation:

$$
C_{l}=\frac{2 \pi a}{(l+1 / 2) T_{l}}
$$

where $a$ is the radius of the Earth. Only the fundamental mode of Love and Rayleigh waves is considered. The phase velocity perturbation at a given period $(\Delta C / C)_{T}$ is obtained from

$$
\left(\frac{\Delta C}{C}\right)_{T}=-\frac{C}{U}\left(\frac{\Delta T}{T}\right)_{l} .
$$

In this equation the group velocity $U$, the reference phase velocity $C$, and the period $T$ have been taken from the preliminary reference Earth model (PREM) values [Dziewonski and Anderson, 1981].

A first test consists of computing the average periods of several eigenmodes from 3SMAC and comparing them with the predictions of the anisotropic version of PREM. Figure 1 depicts the differences for 22 eigenmodes periods between PREM predictions and what is obtained by averaging the local periods of 3SMAC. Our model overestimates the periods for Love modes by $2 . \%$ for $l$ larger than 100 and underestimates the periods by $-0.5 \%$ at most for Rayleigh waves. This difference in behavior between Love and Rayleigh waves partly comes from the assumed isotropy of the model. It is known since the work of Anderson [1966] that only an anisotropic upper mantle can reconcile both data sets. The misfits in eigenmode periods between 3SMAC and the anisotropic PREM are comparable with those be-

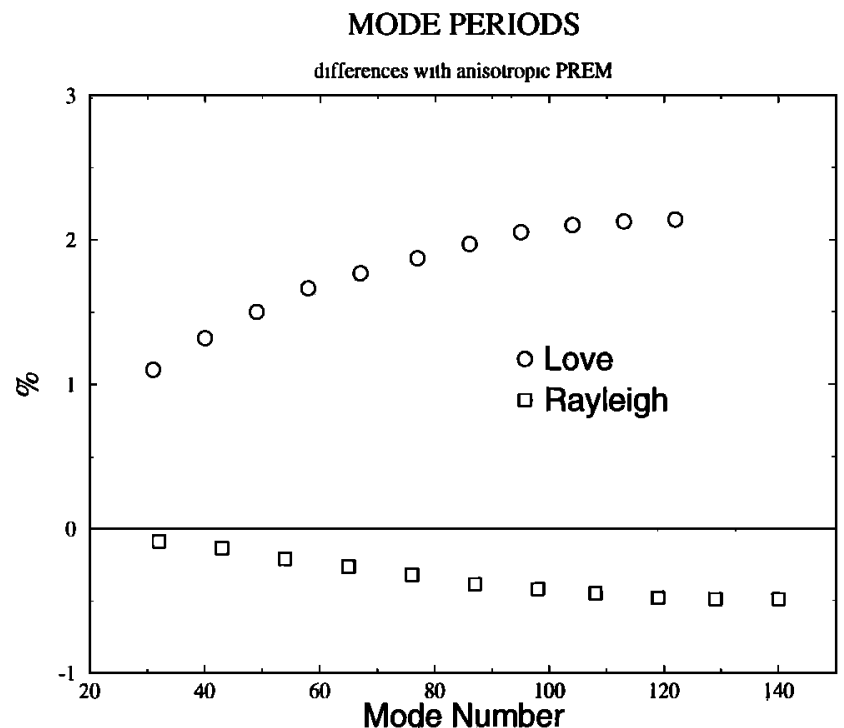

Figure 1. Average periods of 22 eigenmodes predicted by $3 \mathrm{SMAC}$ in percent with respect to PREM values. These periods are up to $2 \%$ larger than anisotropic PREM values for Love waves. tween the isotropic and the anisotropic PREM models.

Figure 2 depicts the phase velocity lateral variations at different periods, predicted by 3SMAC for Love waves at 77 and $110 \mathrm{~s}$ (Figures $2 \mathrm{a}$ and $2 \mathrm{~b}$ ) and for Rayleigh waves at 110 and $200 \mathrm{~s}$ (Figures $2 \mathrm{c}$ and $2 \mathrm{~d}$ ). The four maps have been drawn without contouring or smoothing to show the actual discretization of 3SMAC. The amplitude is in percent with respect to the average phase velocity. Notice the change of scale for the Rayleigh phase velocity at $200 \mathrm{~s}$ (Figure 2d). From Figure 2a to Figure 2d there is an increase in sensitivity of the surface waves to larger depths. There is therefore a decrease in amplitude of the anomalies, as 3SMAC does not include large deep-seated anomalies that could be unambiguously inferred from geodynamics. The largest contrast between fast and slow anomalies is between ridges and cratons for Love waves at 77 $\mathrm{s}$ and between hotspots and slabs for Rayleigh waves at $200 \mathrm{~s}$. Although the amplitudes of these predictions roughly agree with large-scale tomography imaging, the lateral velocity contrasts are larger, and the resolution is better than what is usually obtained in real tomographic experiments.

\section{A Tomographic Experiment}

As 3SMAC predicts phase velocity amplitudes in relative agreement with observations, a synthetic tomography experiment performed using 3SMAC should be indicative of the possible problems associated with an inversion. Using the exact ray coverage of $M \& T$ data set, we integrate along the ray path the seismic slowness anomalies, and then we try to recover the pattern and amplitude of the initial 3SMAC anomalies. The degradation due to the inversion of synthetic data can be considered as indicative of what may exist when real data are used.

From a discrete data set of surface wave observations at a given period, a continuous map of phase velocity can be computed under certain assumptions. For example, various tomographic models have been obtained by expanding the velocities on a limited set of spherical harmonics [e.g., Nakanishi and Anderson, 1984a, b; Woodhouse and Dziewonski, 1984]. We choose a different method derived from what has been used by M\&T. Following the framework given by Tarantola and Valette [1982], we assume that the velocity variations have a requested smoothness. Mathematically, the problem to solve is to invert for a continuous slowness function $m=1 / C(T, \theta, \phi)$ (the "model"), knowing a set of slowness anomalies $d_{i}=1 / C_{\Delta_{i}}(T)$ (the "data") measured along a finite number of paths $\Delta_{i}$. Model and data are related through the linear functional expressed by equation (1), $d=G(m)$. The solution in the least squares sense minimizes the quantity $I(m)$ such that

$$
\begin{aligned}
I(m)=(d-G(m)) C_{d d}^{-1}(d-G(m)) & \\
& +\left(m-m_{0}\right) C_{m m}^{-1}\left(m-m_{0}\right),
\end{aligned}
$$

and is given by 


\section{LOVE PHASE VELOCITY at 77 s (3SMAC)}

(a)

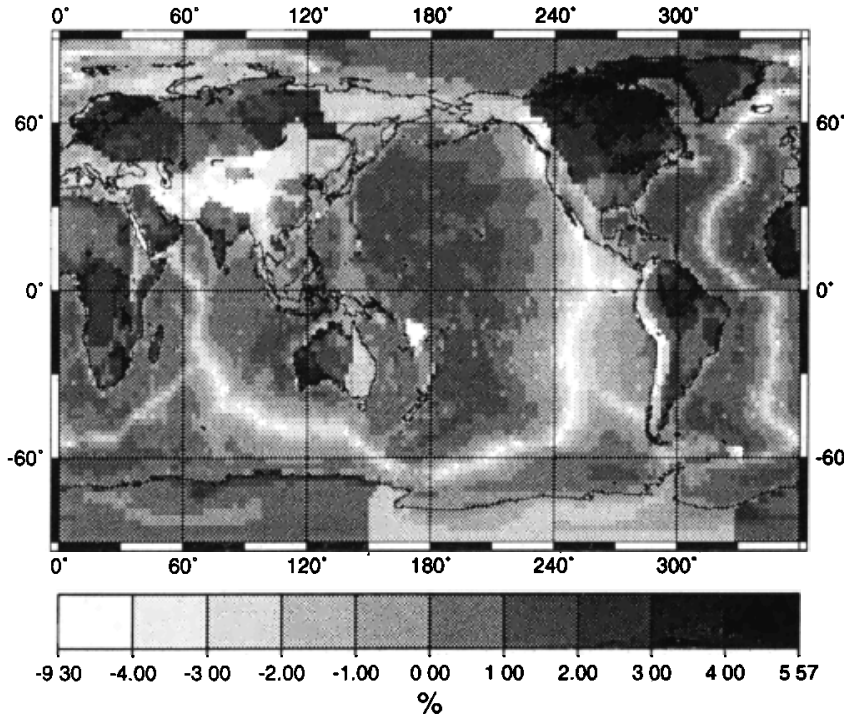

RAYLEIGH PHASE VELOCITY at 110 s (3SMAC)

(c)

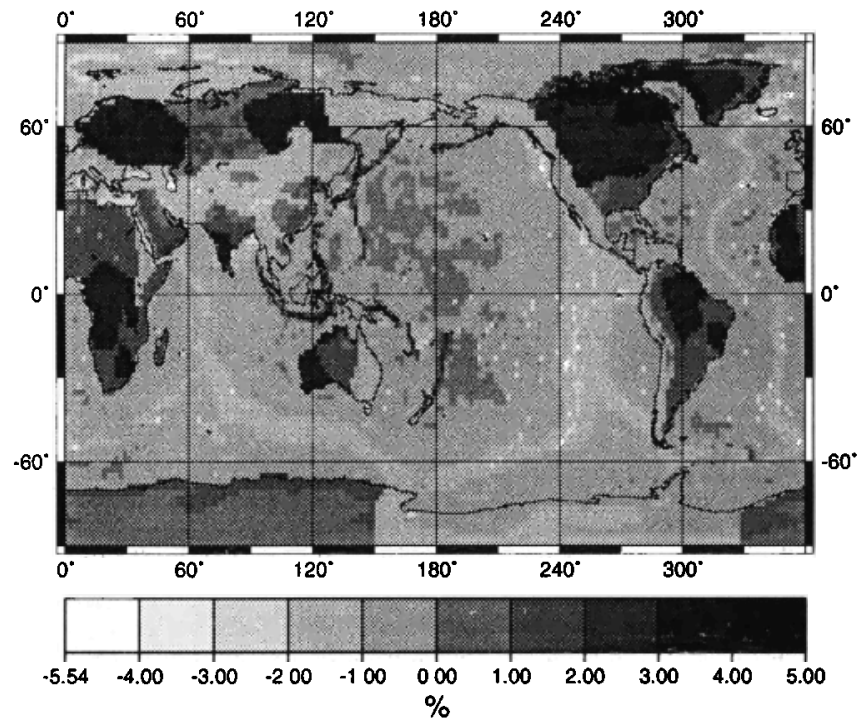

LOVE PHASE VELOCITY at $110 \mathrm{~s}$ (3SMAC)

(b)

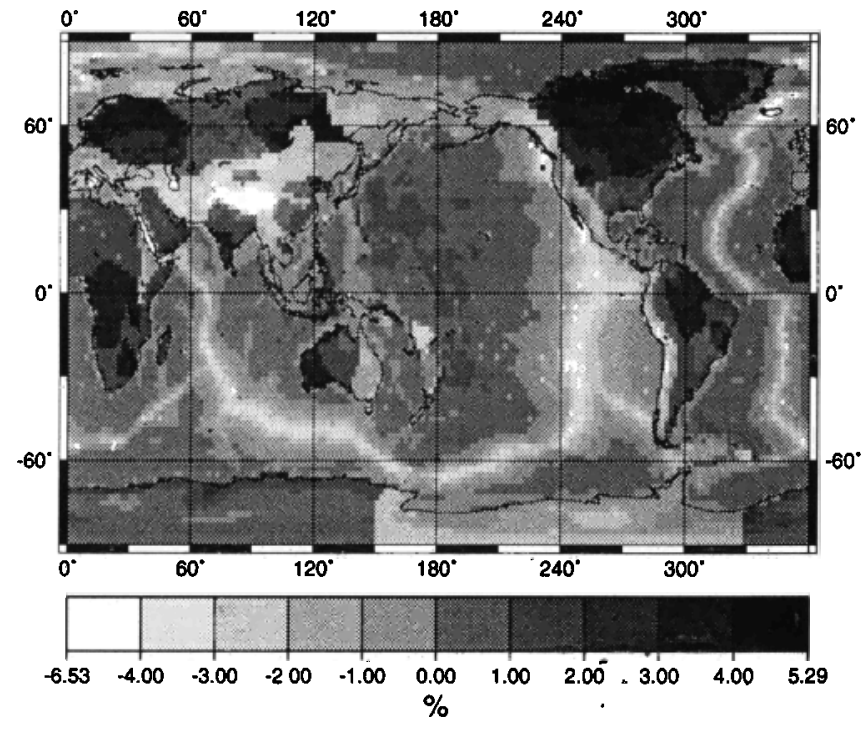

RAYLEIGH PHASE VELOCITY at 200 s (3SMAC)

(d)

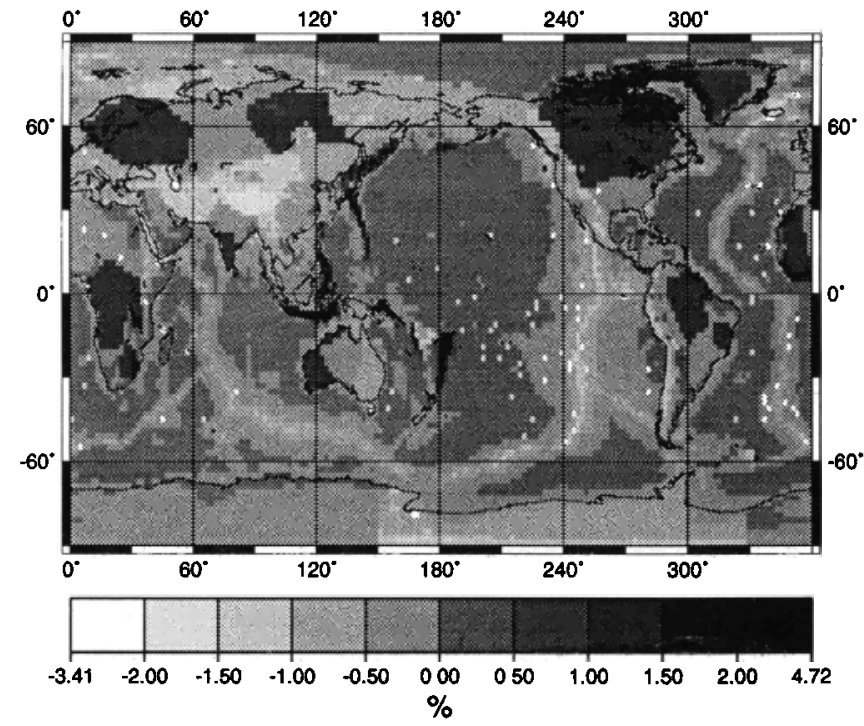

Figure 2. Phase velocities of different surface waves predicted by 3 SMAC, for Love waves at (a) $77 \mathrm{~s}$ and (b) $110 \mathrm{~s}$, for Rayleigh waves at (c) $110 \mathrm{~s}$ and (d) $200 \mathrm{~s}$. These four maps have been chosen to illustrate the sensibility of 3SMAC to surface waves sampling deeper and deeper parts of the Earth. The peak to peak amplitude decreases from around $10 \%$ in Figure $2 a$, to $5 \%$ in Figures $2 \mathrm{~b}$ and $2 \mathrm{c}$, and to only $2 \%$ in Figure $2 \mathrm{~d}$. The discretization of 3SMAC $\left(2^{\circ} \times 2^{\circ}\right)$ is clearly visible by the absence of any smoothing in the maps.

$$
\begin{aligned}
& m=m_{0} \\
& \quad+C_{m m} G^{t}\left(C_{d d}+G C_{m m} G^{t}\right)^{-1}\left(d-G\left(m_{0}\right)\right),
\end{aligned}
$$

where $m_{0}$ is the a priori model and $C_{m m}$ and $C_{d d}$ are the covariance matrices for model and data, respectively. The a priori covariance matrix for the model can be chosen as

$$
C_{m m}=\delta^{2} m_{0} \exp \left(-\frac{\Delta^{2}}{2 \Delta_{0}^{2}}\right),
$$

where $\delta m_{0}$ is a standard deviation, $\Delta$ is the angular distance between two points, and $\Delta_{0}$ a correlation angle that controls the smoothness of the inverted model. Here, $\delta m_{0}$ is chosen as $1 \%$ of the PREM value, but this value does not appear crucial for our results. The data 
covariance matrix is assumed diagonal, and its diagonal elements take the form $\delta^{2} d_{\imath}=\left(\delta t / \Delta_{i}\right)^{2}$. We choose $\delta t=13 \mathrm{~s}$ to take into account the error in the phase velocity measurement, mostly due to a sampling rate, which is generally $10 \mathrm{~s}$.

A first problem is to compute the inverse matrix in the right-hand side of equation (4) (the term corresponding to $\left.\left(C_{d d}+G C_{m m} G^{t}\right)^{-1}\right)$. However, assuming that the correlation angle $\Delta_{0}$ is smaller than both the ray length $\Delta_{i}$ and the average distance between rays, one can show that $G C_{m m} G^{t}$ is diagonal and that its diagonal elements are closely approximated by

$$
\left(G C_{m m} G^{t}\right)_{\imath 2} \sim \delta^{2} m_{0} \sqrt{2 \pi} \frac{\Delta_{0}}{\Delta_{i}} .
$$

This approximation neglects all correlations between rays. This is generally correct except in a few cases, when two earthquakes and a station or one earthquake and two stations are on the same great circle.

A second problem is to apply a Gaussian smoothing on a sphere (the term corresponding to the effect of $C_{m m} G^{t}$ ). Instead of performing a time-consuming convolution in space, we work in the spectral domain. We perform a low-pass filtering in the spherical harmonic domain corresponding to a multiplication of the harmonic coefficients:

$$
C_{m m} G^{t} \longmapsto \delta^{2} m_{0} e^{-(l+1 / 2)^{2} \Delta_{0}^{2} / 2} .
$$

This is indeed the analogue for the sphere of the Fourier transform of the expression (6).

The inversion method is thus the following. The use of PREM allows us to compute the a priori uniform slowness model $m_{0}$ for the chosen period. For each path $i$ a misfit $d_{2}-G(m)_{2}$ is computed. This misfit is weighted by $S_{i}$ where

$$
S_{i}=\delta^{2} d_{i}+\delta^{2} m_{0} \sqrt{2 \pi} \frac{\Delta_{0}}{\Delta_{i}} .
$$

These weighted misfits are then added in all the $2^{\circ}$ by $2^{\circ}$ cells crossed by the rays. Following this procedure, we obtain a $2^{\circ}$ by $2^{\circ}$ degree map of estimated slownesses. This map is low-pass filtered. The new slowness model $m(\theta, \phi)$ is then used iteratively until convergence is achieved and therefore until the minimization of $I(\mathrm{~m})$ is reached. To avoid aberrant data, all data having an a posteriori residue larger than $2 \delta d_{b}$ are suppressed from the data file (only a few percent of M\&T data are effectively thrown out), and a new inversion is performed.

The choice of the correlation angle $\Delta_{0}$ is crucial. Clearly, if we want enough redundancy, a large correlation angle is requested. However, the choice of a large correlation angle will give us a smooth and maybe unrealistic model. Moreover, it will not take full advantage of the method that uses a local smoothing criterion instead of a finite set of expansion functions. Indeed, as the ray path density varies from place to place, a resolution corresponding to short wavelengths can be obtained locally in highly sampled areas.
The problem of chosing $\Delta_{0}$ is due to the difficulty of computing the a posteriori errors of the inverted model. Theoretically, the choice of a correlation angle that is too small should lead to the computation of a model having large errors. Instead of computing the a posteriori covariance matrix corresponding to the inverse problem, we prefer a purely empirical method using 3SMAC as a touchstone for the quality of the inversion. A synthetic data set using $3 \mathrm{SMAC}$ is computed, and noise having the statistical variance $\delta t / \Delta$ is added. This data set is processed by the inversion program for various correlation lengths, and both the distance to data (first term of the right-hand side of equation (4)) and the distance to 3SMAC are computed. Whereas decreasing the correlation length always leads to a better fit to the synthetic data, it does not systematically improve the distance to 3SMAC. This means that with the use of a $\Delta_{0}$ that is too small the inversion explains noise but not signal.

Figure 3 summarizes the results of the synthetic tests using 3SMAC. Practically, we tested the correlation angles of $3^{\circ}, 4.5^{\circ}, 6^{\circ}, 7.5^{\circ}, 9^{\circ}$, and $12^{\circ}$. For various periods we plot the best variance reduction that has been obtained as a function of the corresponding correlation angle. This variance reduction is defined by

$$
v_{r}(T)=1-\frac{\int\left(C_{3 S}(T, \theta, \phi)-C_{I}(T, \theta, \phi)\right)^{2} d S}{\int C_{3 S}^{2}(T, \theta, \phi) d S},
$$

where $C_{3 S}$ and $C_{I}$ are the initial (3SMAC) and inverted local phase velocities. The integrals are performed on the whole sphere.

For Love and for short-period Rayleigh waves a small correlation angle of $3^{\circ}$ gives the best results. However,

\section{SMAC INVERSION}

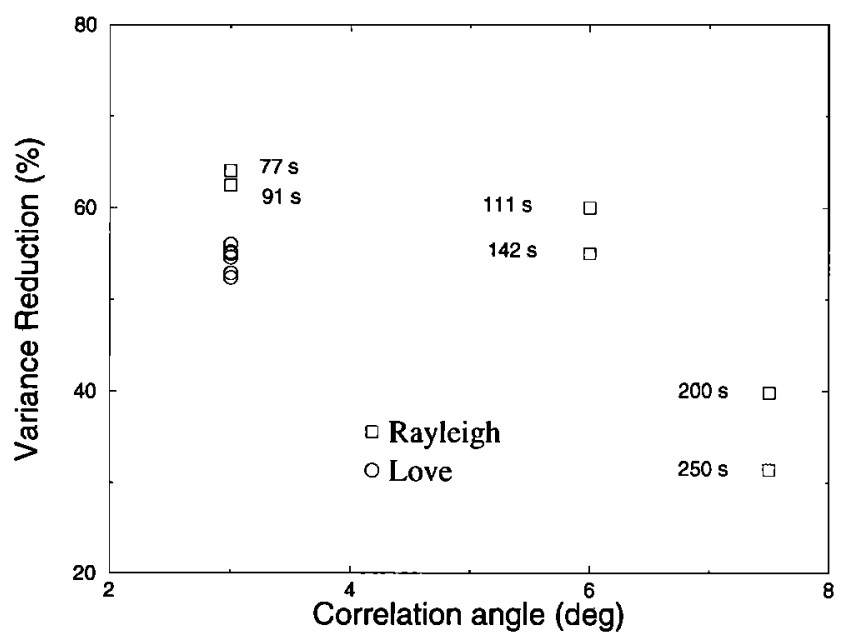

Figure 3. Best variance reductions in percent obtained by our inversion method tested with 3SMAC synthetics as a function of the corresponding correlation angle in degrees. As the period of surface wave increases, the variance reduction decreases. For Love waves, the results for all periods are grouped around $55 \%$. For short periods a better signal to noise ratio allows us to choose a small correlation angle. 
for Rayleigh waves at longer periods the correlation length has to be increased. Indeed, the velocity perturbations predicted by 3SMAC decrease with period, whereas the noise remains constant. The choice of a largely unsuitable correlation length decreases the variance quite a bit. As examples, for Rayleigh waves at 77 $\mathrm{s}$ the variance reduction goes from $64 \%$ with $\Delta_{0}=3^{\circ}$ to $58 \%$ with $\Delta_{0}=12^{\circ}$, for Rayleigh waves at $250 \mathrm{~s}$ the variance reduction reaches a maximum of $32 \%$ at $\Delta_{0}=7.5^{\circ}$ but decreases to $16 \%$ or $29 \%$ for $\Delta_{0}=3^{\circ}$ or $\Delta_{0}=12^{\circ}$, respectively. Because of the very short wavelength content of $3 \mathrm{SMAC}$ one notices that even the best variance reductions correspond to an explanation of only about $60 \%$ of the data.

\section{Phase Velocity Maps}

A tomographic method closely related to our procedure has been shown by Ho-Liu et al. [1989] to be equivalent to that used by Clayton and Comer [1983] in body wave travel time tomography. The only difference is that a common simultaneous iterative reconstruction technique (SIRT) method introduces an empirical damping parameter which naturally arises from the last term of equation (8). However, Trampert [1990] noticed that the iterative solution does not correspond to the exact solution of a linear problem given without iterations by equation (5). This is certainly right. We also agree that we are cheating in some respects (approximation of a matrix in equation (7), approximation of a Gaussian convolution in equation (8), approximation of an inverse matrix in equation (9)) to handle equation (5). At any rate, it should be clear that performing synthetic tests is much easier than working with real data; at least we know what should be obtained. The tomographic procedure we use and the parameters we define have been chosen to give the best results in recovering the heterogeneities of 3SMAC. We leave the possibility to other seismologists to test their programs against 3SMAC and to check whether a better job can be done.

A simple test consists of inverting a simple perturbation represented by a pure spherical harmonic. We make use of two different data sets for Love wave paths, the data set of M\&T (2184 rays) and that of Zhang and Tanimoto [1993] (8981 L1 rays), in order to see the effect of densifying the path coverage. The perturbation has a $2 \%$ rms amplitude. Noise corresponding to a statistical time error of $13 \mathrm{~s}$ is added to compute synthetic phase velocities. For an anomaly described by a degree lower than 10 the inverted pattern is rather closely retrieved. To show that a signal can be obtained even at much shorter wavelengths, we perform the exercise with degree 15 , order 7 , and degree 22 , order 11 . For these harmonics the peak to peak velocity variations corresponding to a $2 \% \mathrm{rms}$ amplitude are about $11 \%$. These values are fairly large but not totally unrealistic for Love waves.

Figure 4 depicts the patterns obtained after inversion of the two spherical harmonics with a correlation length of $3^{\circ}$. At degree 15 (Figures $4 a$ and $4 b$ ) the data coverage of M\&T seems to be pushed toward its limit. A much better result is obtained by using 8981 data, particularly in the northern hemisphere, whereas strong biases are present in the southwest Pacific and around Antartica. The variance reductions achieved by the inversions corresponding to the two maps are $38 \%$ and $65 \%$ for the small and large data sets, respectively. Even at degree 22 (Figures $4 \mathrm{c}$ and $4 \mathrm{~d}$ ) a signal is still clearly detectable, but surprisingly, the resolution obtained from the small data set does not appear much poorer than that obtained from the large data set except under North America. The variance reductions are now $24 \%$ and $28 \%$, respectively. We will see later that for velocity anomalies of even degrees (e.g., of degree 22 ), the two path coverages lead to somewhat similar resolution, whereas the path coverage that includes only L1 paths of Zhang and Tanimoto [1993] yields a better resolution for anomalies of odd degrees (e.g., of degree 15).

The next two figures depict for the same periods as in Figure 2 the phase velocity maps obtained after inversion of 3SMAC synthetics (Figure 5) and of M\&T observations (Figure 6). The comparisons between the maps of Figure 2 and Figure 5 give a visual indication of the importance of the smoothing that has been introduced by the inversion. The correlation lengths are $3^{\circ}$ for the Love waves and $6^{\circ}$ and $7.5^{\circ}$ for Rayleigh waves at 110 and $200 \mathrm{~s}$, hence in agreement with Figure 3 . The comparisons between synthetics (Figure 5) and observations (Figure 6) are satisfactory for the four maps. Not only the patterns but also the amplitudes are correctly predicted. This is the case even though 3SMAC has been built totally independently from surface wave observations. Absolutely no parameter inversion has been done to improve the fit of our model with observations.

The 3SMAC model generally satisfies the observations, but the predictions are better for Love waves than Rayleigh waves, as expected, and some discrepancies are clearly visible. First, some structures are not predicted with their correct amplitudes. They are somewhat overestimated for long-period Rayleigh waves and over cratons at all periods. These two last observations are surprising: They suggest that $3 \mathrm{SMAC}$ includes anomalies that are too strong and/or too deep, although the a priori lithosphere is already rather thin, as we assumed that the temperature under cratons reaches the adiabat at only $300-\mathrm{km}$ depth. Second, some anomalies revealed by seismic observations are not predicted by $3 \mathrm{SMAC}$, as they do not have a clear surface expression. These are, for example, the low velocities around the Red Sea and above Southeast China. Of course, 3SMAC includes a strip of oceanic lithosphere in the Red Sea and a thin lithosphere under the Afars and toward the African High Plateaus. However, the upper mantle seems much slower indeed than what we guessed from the surface. Over Southeast China the anomalous slow structure is also surprising. The 3SMAC model predicts a slow root associated with the very thick crust of the Tibetan plateau, west of the real location of a 
INVERSION of HARMONICS L=15 M=7 (2184 data)

(a)
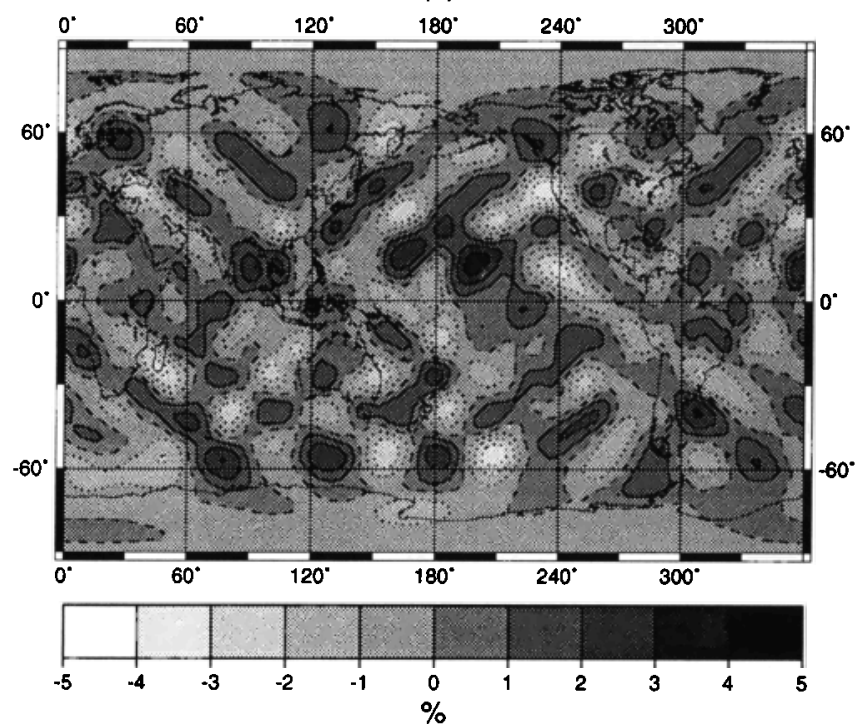

INVERSION of HARMONICS $L=22 M=11$ (2184 data)

(c)

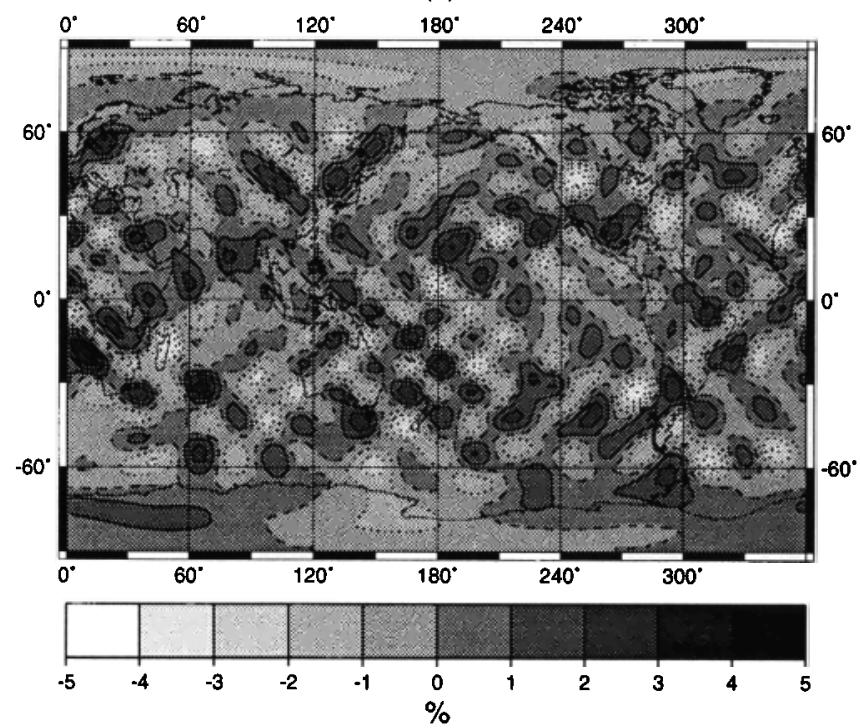

INVERSION of HARMONICS $L=15 \mathrm{M}=7$ (8981 data)

(b)

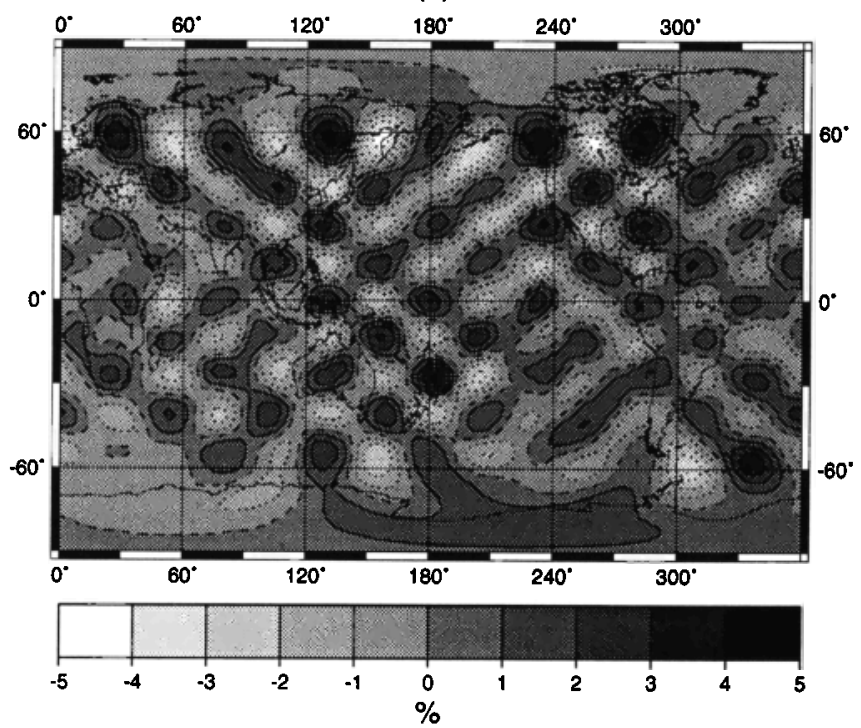

INVERSION of HARMONICS L=22 M=11 (8981 data)

(d)

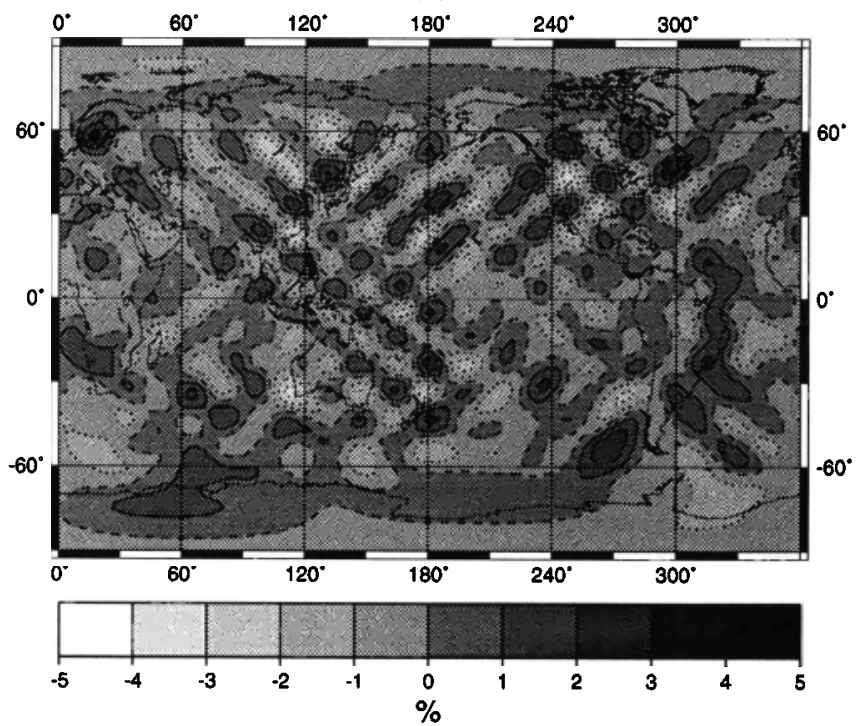

Figure 4. Synthetic inversion of phase velocity patterns corresponding to spherical harmonics (a, b) $l=15, \mathrm{~m}=7$ and (c, d) $l=22 \mathrm{~m}=11$. The peak to peak amplitude of the original signal is around $11 \%$. The path coverage of 8981 rays gives better results in the northern hemisphere than that of 2184 rays, for $l=15$. For $l=22$ the input signal is still visible, but the distortion is visually comparable for the two data sets.

slow anomaly. This anomaly corresponds to a geological setting that we included in the "stable area" class of our regionalization. The comparison of observations with 3SMAC suggests that the undeformed old crust may be lying above a rejuvenated lithosphere, maybe reheated during its eastward extrusion linked to the indentation of the Eurasian Plate by India [Tapponnier et al., 1982]. Greenland also appears different from what has been predicted by 3SMAC. In many regionalizations [e.g., Jordan, 1981], Greenland is included within the same cratonic area as the Canadian Shield. It is, however, less fast. The opening of the Atlantic has probably affected its lithosphere to a larger extent than what can be deduced from the geological observations in the few locations uncovered with ice. For long-period Rayleigh waves, there is a disagreement in the location of lowvelocity zones under the Pacific plate. The East Pacific Rise is offset westward with respect to what is predicted by $3 \mathrm{SMAC}$, and a large low-velocity anomaly is present between New Zealand and Antartica. 
LOVE PHASE VEL. at $77 \mathrm{~s}$ (Inverted 3SMAC)

(a)

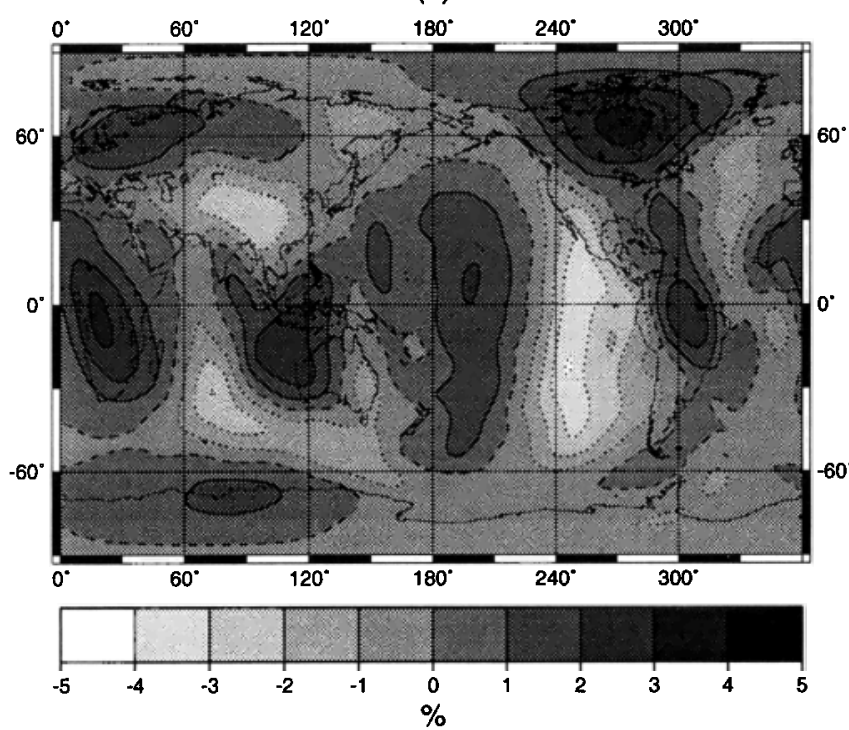

RAYLEIGH PHASE VEL. at 110s (Inverted 3SMAC)

(c)

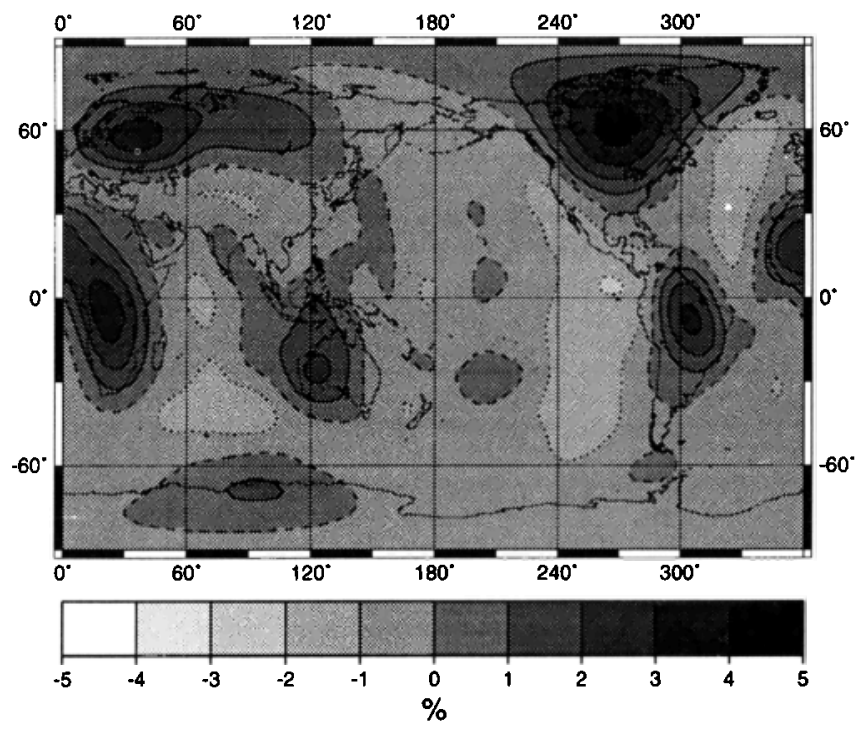

LOVE PHASE VEL. at $110 \mathrm{~s}$ (Inverted 3SMAC)

(b)

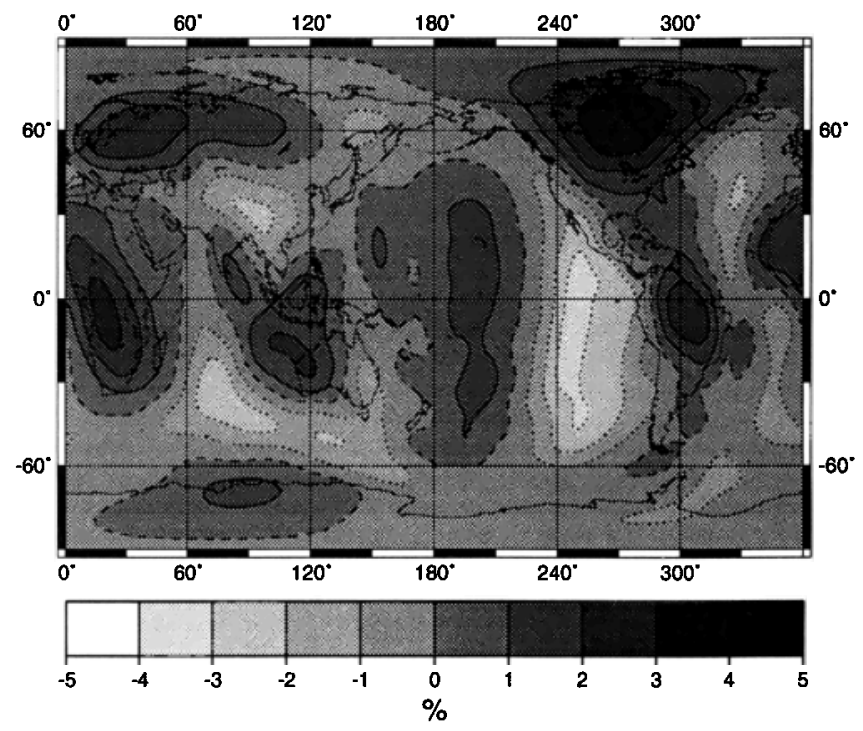

RAYLEIGH PHASE VEL. at 200 s (Inverted 3SMAC)

(d)

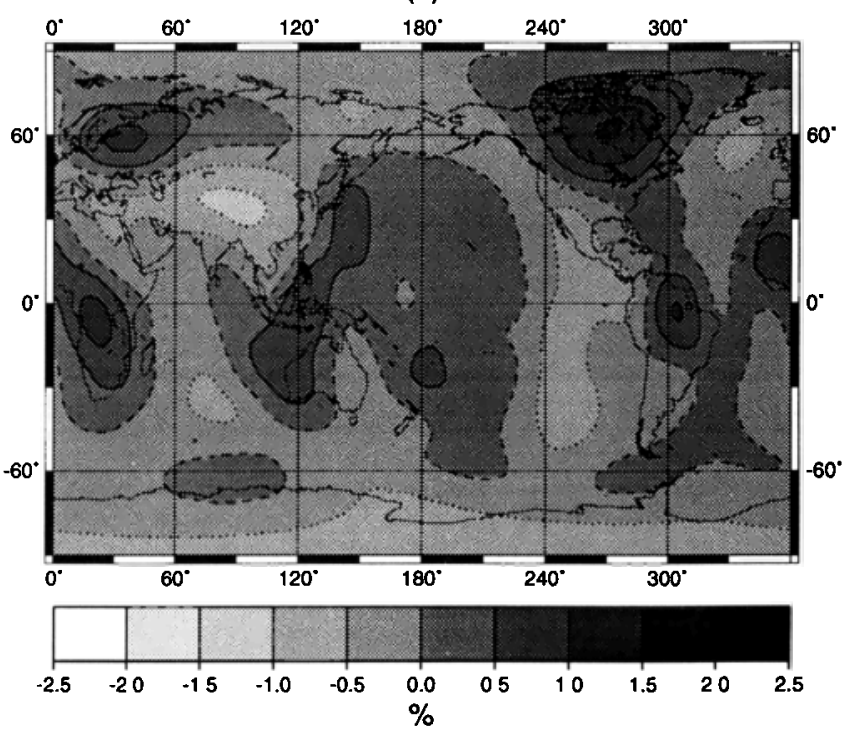

Figure 5. Phase velocities retrieved by tomographic inversion of 3SMAC synthetics. M\&T ray paths are used. These maps should be the same as those of Figure 2 for a perfect inversion. We use correlation angles of $(\mathrm{a}, \mathrm{b}) 3^{\circ}$, (c) $6^{\circ}$, and (d) $7.5^{\circ}$.

\section{Correlations and Spectra}

Figure 7 depicts the correlations between the velocity perturbations directly predicted by $3 \mathrm{SMAC}$ and those inverted from 3SMAC synthetics by using M\&T ray coverage, for Love and Rayleigh waves at $110 \mathrm{~s}$. This plot quantifies the correlations between Figures $2 b$ and $5 \mathrm{~b}$ and between Figures $2 \mathrm{c}$ and $5 \mathrm{c}$. They have been computed up to degree 50. A thin dotted line depicts the $95 \%$ confidence level. All the correlation coefficients for degrees less than 19 lie above this line. This finding proves that the ray coverage is dense enough to re- cover the heterogeneity pattern down to a wavelength of some $2000 \mathrm{~km}$. The results obtained by using the 3610 Rayleigh wave paths (dotted line) are only slightly better between degrees 6 and 12 than those obtained with the reduced set of 2184 Love paths (solid line). In fact, two polarizations have been recorded for much of the Rayleigh data. Therefore the numbers of different paths in M\&T Love and Rayleigh are basically the same.

Figure 8 is similar to Figure 7 , but the correlations are now computed between inverted 3SMAC synthetics and inverted data. The solid line thus corresponds to the correlations between Figures $5 \mathrm{~b}$ and $6 \mathrm{~b}$, and the 
LOVE PHASE VEL. at $77 \mathrm{~s}$ (Observed)

(a)

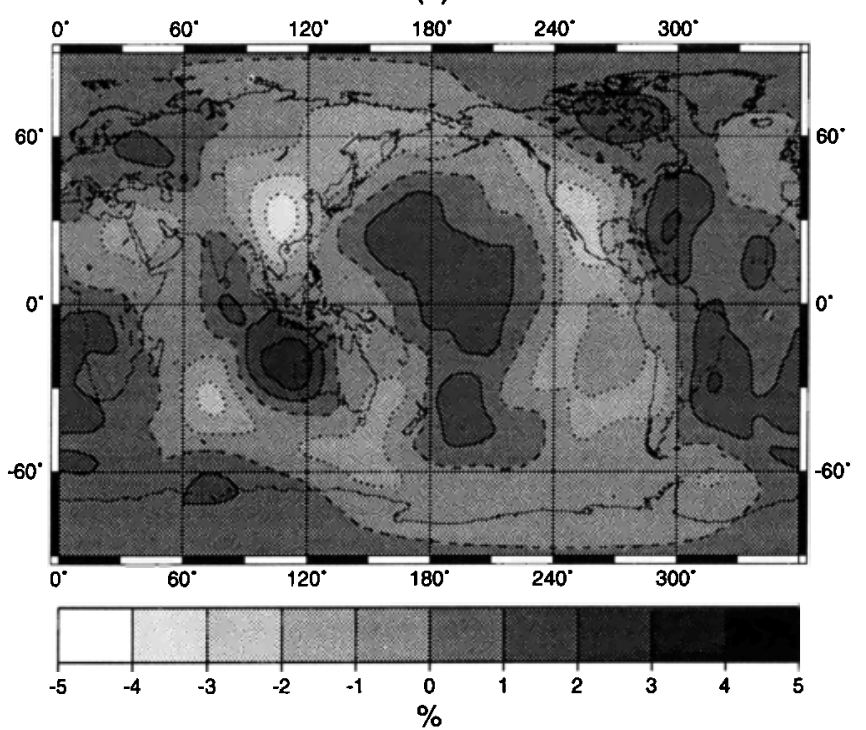

RAYLEIGH PHASE VEL. at $110 \mathrm{~s}$ (Observed)

(c)

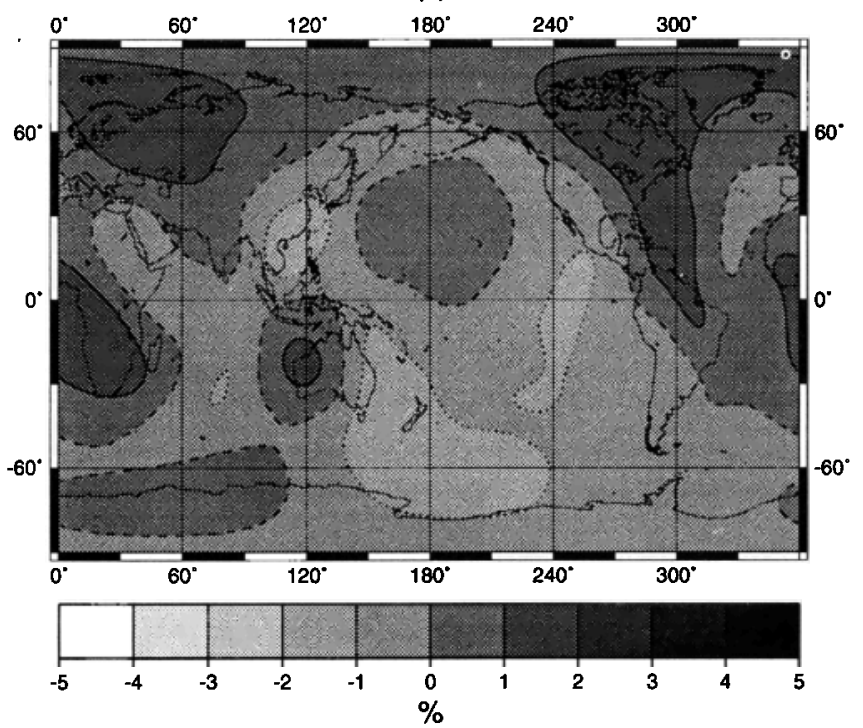

LOVE PHASE VEL. at $110 \mathrm{~s}$ (Observed)

(b)

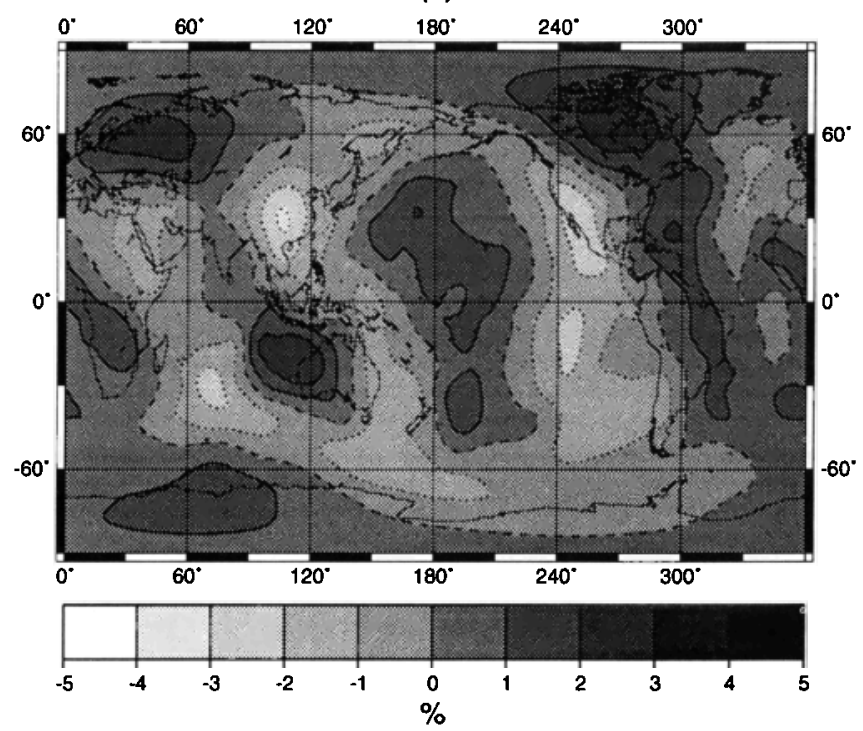

RAYLEIGH PHASE VEL. at 200 s (Observed)

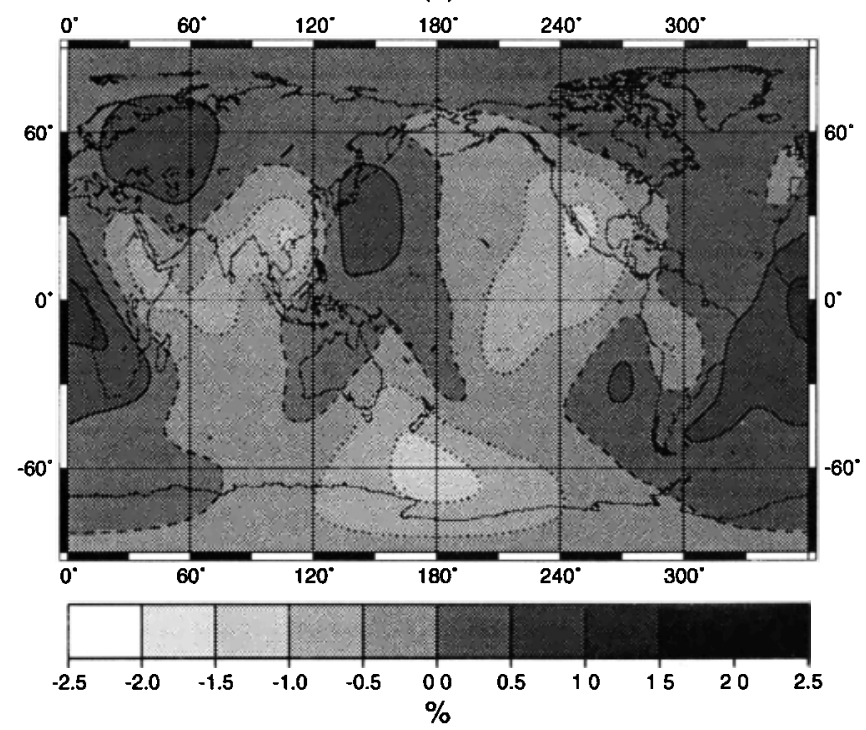

Figure 6. Phase velocities retrieved by tomographic inversion of the M\&T data set. These maps have to be compared with the previous ones and generally agree with them in both pattern and amplitude.

dotted line to the correlations between Figures $5 c$ and 6c. Although some low degrees are below the $95 \%$ confidence level, the average level of correlation is high: up to degree $50,92 \%$ and $78 \%$ of the correlations for Love and Rayleigh waves, respectively, are above the $95 \%$ confidence level. This figure is computed for a period of $110 \mathrm{~s}$. However, it is representative of the results obtained from $70 \mathrm{~s}$ to $250 \mathrm{~s}$. The increase of correlation at high degrees is surprising. It due to the path distribution rather than to the velocity anomalies themselves.

To check whether amplitudes are also correctly retrieved by the inversion, the spectral amplitudes of the a priori 3SMAC heterogeneities (solid line) as well as those obtained by the inversion of 3SMAC synthetics (dotted line) and by the inversion of real data (dashed line) are depicted in Figure 9 for three different periods. The spectral amplitude $A(l)$ of a given function $A(\theta, \phi)$ is simply defined by

$$
A(l)=\sum_{l=-m}^{l=m} a_{l m}^{2}
$$

where $a_{l m}$ are the coefficients of the expansion of $A(\theta, \phi)$ 


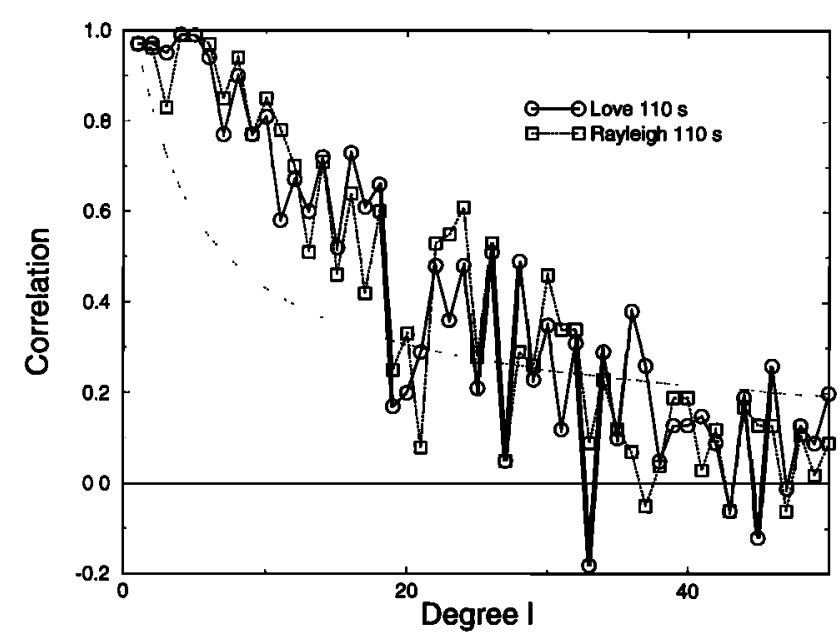

Figure 7. Correlations between 3SMAC velocities and the results of inversions using synthetics for Love and Rayleigh waves at $110 \mathrm{~s}$. All the correlation coefficients up to $l=19$ and many of them between $l=20$ and $l$ $=50$ lie above the $95 \%$ confidence level (dotted line).

on the spherical harmonics basis normalized following the convention of Stacey [1977]. Two comments can be made: First, the spectra of observations and inverted 3SMAC are rather similar (dotted and dashed lines); and second, the inversion strongly biases the real spectra (dotted and solid lines) for degrees larger than 10. Even at very low degree the agreement between direct and inverse coefficients is far from perfect: For Rayleigh at $110 \mathrm{~s}$, for example, the recovered degree 3 accounts for only $53 \%$ of the original signal (correlation of 0.82 and overestimation of the amplitude by a factor 1.2). For degrees larger than 6 or 8 the amplitudes are greatly underestimated by the inversion. Whereas the real spectrum roughly decreases with $l^{-\alpha}$ with $\alpha$ between 0.8 and 0.9 , the inversion predicts a $l^{-\beta}$ depen-

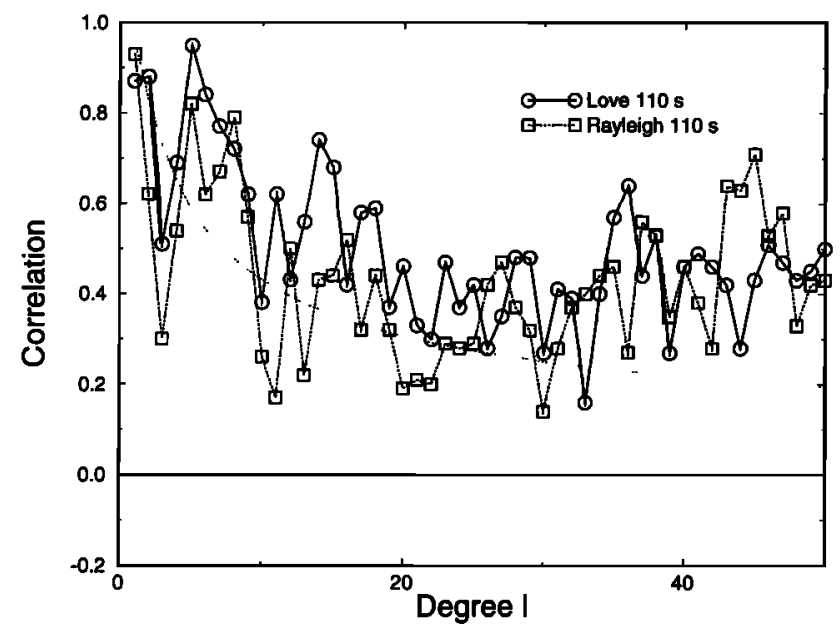

Figure 8. Correlations between the results of inversions using 3SMAC synthetics and using real observations for Love and Rayleigh waves at $110 \mathrm{~s}$. Although these coefficients are on average lower than those between 3SMAC and inverted 3SMAC (Figure 7), most of them are significant. dence of the heterogeneity spectrum with $\beta$ between 1.8 and 2.5. Of course, the slope of the spectra decreases when the correlation length decreases (from $7.5^{\circ}$ (bottom), to $6^{\circ}$ (middle) and to $3^{\circ}$ (top)). However, even for degrees corresponding to wavelengths much larger than the correlation lengths, say, for the first 12 degrees, the inverted spectrum significantly differs from the input. The inversion clearly favors the even degrees and damps the odd ones. This effect, already suspected in different tomographic models, was explained by Mochizuki [1993]. For the shortest path the resolving sensibility of Earth lateral heterogeneity by line integrals decreases as the inverse square root of $l$. For the longest paths, a strong damping of the odd degrees superimposes onto the decrease of the sensitivity with $l$. Our numerical test clearly illustrates this smoothing effect of tomographic inversion. Decreasing the correlation length does not solve the problem; on the contrary, we have seen that it decreases the quality of the inversion.

We tried different ways of circumventing the poor amplitude restitution of the inversion. We decreased the weight of the long paths (R2 or L2) with respect to R1

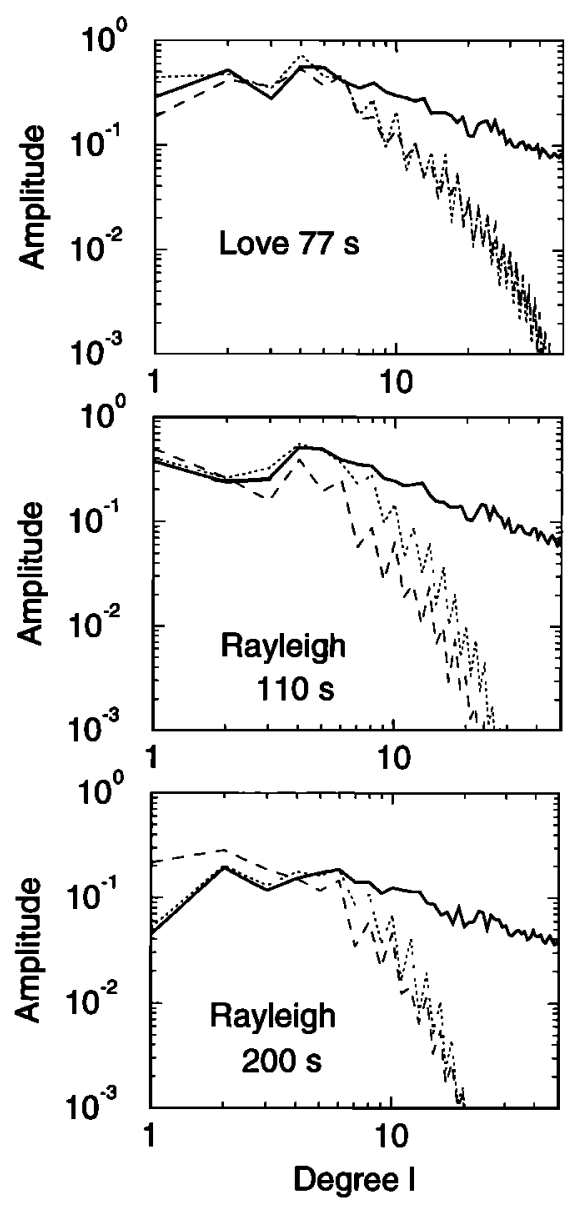

Figure 9. Amplitude spectra of 3SMAC (solid line) together with those obtained by inversion of 3SMAC synthetics (dotted line) and of real data (dashed line). The two inverted data sets have rather similar spectra characterized by a rapid decrease and an oscillatory behavior. They both drop with increasing degrees much faster than what is predicted by 3SMAC. 

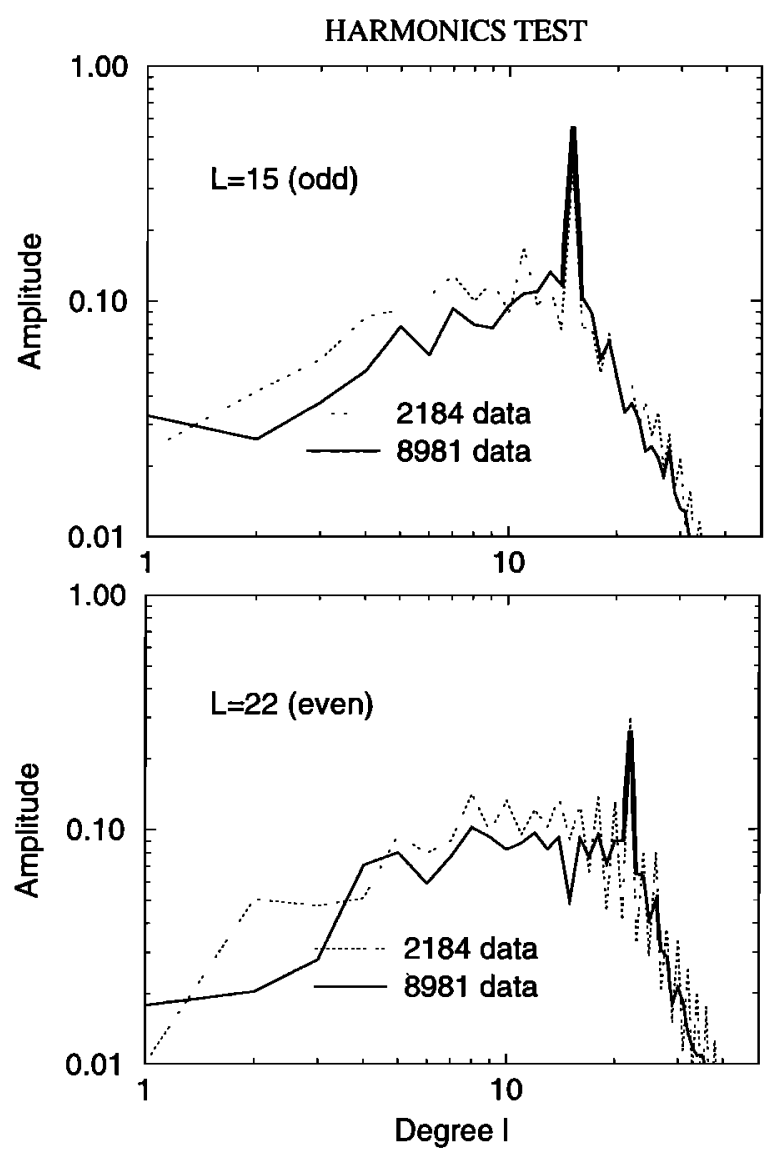

Figure 10. Amplitude spectra of the four tests of Figure 4 . The input spectra consist of a spike at (top) $l=15$ and (bottom) $l=22$. The spectra have been normalized with respect to the amplitude of the input spike. The dotted line has been obtained with the M\&T data set. Its oscillatory behavior is due to the presence of L2 paths. A slightly better signal to noise ratio is obtained when the $\mathrm{L} 1$ path coverage of Zhang and Tanimoto [1993] is used (solid line).

or L1 paths. This approach dampens the oscillatory behavior of the spectra but decreases the overall quality of the inversion as it effectively decreases the number of data. The consequence of an increase in the number of paths and of the choice of only direct paths is illustrated in Figure 10. It depicts the spectra of the maps resulting from the inversion of pure harmonics (Figure 4). The solid lines are computed with a coverage of 2184 rays ( $\mathrm{L} 1$ and $\mathrm{L} 2$ paths), and the dotted lines are for the $8981 \mathrm{~L} 1$ rays of Zhang and Tanimoto, [1993]. The top diagram corresponds to the inversion of the pure spherical harmonics with $l=15, m=7$, and the bottom is for $l=22, m=11$. The amplitudes have been normalized so that a perfect inversion should have led to zero amplitudes except for a single peak of amplitude 1. The actual inversions lead to peaks at the correct degrees which are perfectly correlated with the observations; for the four inversions the correlations at the input degrees are between 0.95 and 1 . Using only short path data (dotted line) suppresses the oscillatory behavior introduced by the presence of both $\mathrm{L} 2$ paths.

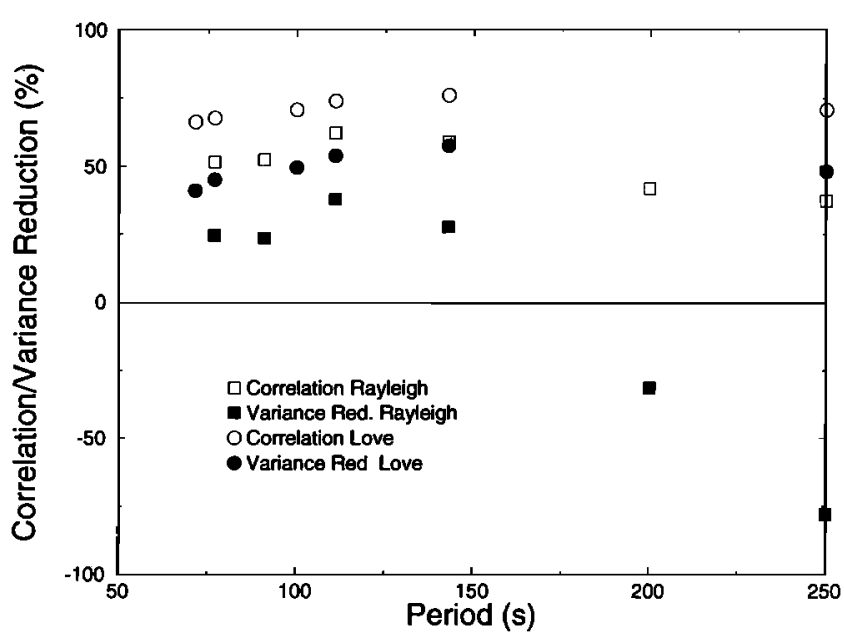

Figure 11. Global correlations between the results of the inversion of 3SMAC synthetics and those of real data (open symbols). The correlations are high and better for Love (circles) than for Rayleigh wave (squares).The corresponding variance reductions are depicted with black symbols. A high variance reduction needs both a high correlation and a correct amplitude. For long-period Rayleigh waves, 3SMAC does not predict the correct amplitude and does not decrease the observation variance.

The recovered amplitudes with respect to the real input signal are only $50 \%$ (degree 15,8981 paths), $30 \%$ (degree 15, 2184 paths) 20\% (degree 22, 8981 paths) and $20 \%$ (degree 22, 2184 paths). Although for odd degrees the large data set with $\mathrm{L} 1$ paths gives somewhat more reliable amplitudes, not much resolution is gained for the even degree. Noise with an amplitude of more than $10 \%$ of that of the input peak appears at various degrees. This noise does not decrease in inverse proportion to the number of data.

We also weighted the a priori model variance by a function of the local path density as suggested by Snzeder [1993]. This weighting penalizes the large excursions of the model in poorly sampled regions, which are often obtained in least squares inversion. However, this does not improve our inversion, because we do not expand the velocity perturbation in spherical harmonics by a least squares method but instead use a local smoothing before the harmonic expansion.

For various Love and Rayleigh waves we compute the phase velocity maps obtained by inversion of observed (M\&T) and synthetic (3SMAC) data. The global correlations and the variance reductions between the two results are displayed in Figure 11. The level of correlation for Love waves is larger than $70 \%$ for all degrees and reaches $50 \%$ for Rayleigh waves, although it decreases to $20 \%$ at larger periods (250 s). For example, the global correlations between Figures $5 \mathrm{a}$ and $6 \mathrm{a}, 5 \mathrm{~b}$ and $6 \mathrm{~b}, 5 \mathrm{c}$ and $6 \mathrm{c}$, and $5 \mathrm{~d}$ and $6 \mathrm{~d}$ are $66 \%, 74 \%, 62 \%$, and $42 \%$, respectively, and 3SMAC explains $41 \%, 50 \%$, $38 \%$, and $-30 \%$ of the observations. Already, Montagner and Jobert [1988], showed that the a priori regionalization led to a similar or a better variance reduction than 
LOCAL EIGENFREQUENCY OS22 L=2 (Smith \& Masters, 89)

(a)

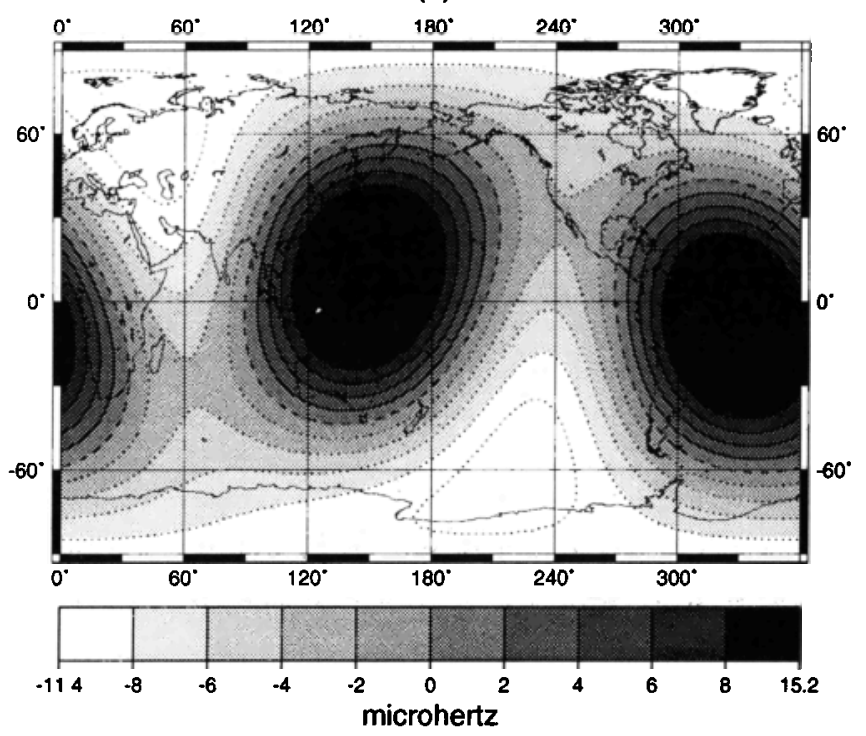

LOCAL EIGENFREQUENCY OS22 L=2 (3SMAC)

(b)

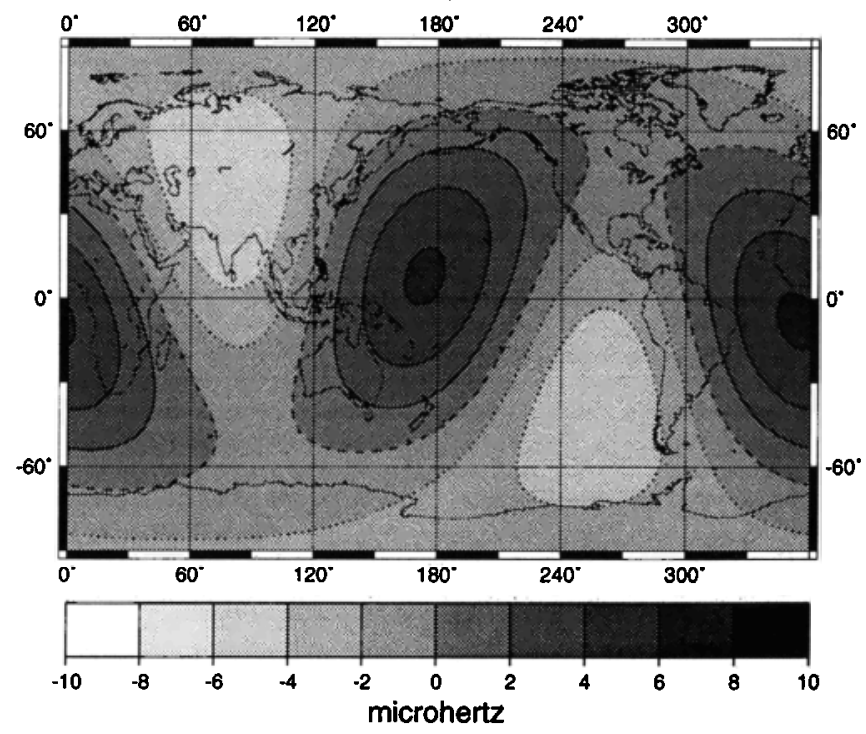

Figure 12. Degree 2 local eigenfrequency anomaly recorded (a) for the mode 0S22 [Smith and Masters, 1989] and (b) that predicted by 3SMAC. The amplitudes are in microhertz. 3SMAC accounts for $42 \%$ of the total observed signal.

the continuous inversion for periods up to $100 \mathrm{~s}$. Thus, except for the long-period Rayleigh waves the amplitudes are correctly predicted, and a significant variance reduction is achieved.

\section{Some Comments on the Lowest Degrees of Mantle Heterogeneity}

One of the first clearly observed velocity anomalies revealed is the so-called degree 2 anomaly [Masters et al., 1982]. This anomaly consists of two roughly equatorial rapid zones located around the West Pacific and Central America. Its pattern dominates the heterogeneities of low degree, and depth inversions locate this anomaly in the mantle transition zone. The most obvious evidence comes directly from observations of normal mode perturbations. This degree 2 anomaly pattern leaps out at you by simply plotting the normal mode perturbations at the pole of the great circle joining an earthquake to a recording station. Computing normal modes in 3SMAC goes beyond the scope of this paper. However, the even degree pattern that we can derive from 3SMAC assuming that the local period can be computed from the vertical column of the seismological parameters should give us a first-order approximation of the real normal mode perturbation. Therefore we use spheroidal modes such as those of degrees 20 to 40 to quantify the importance of deep sources with respect to lithospheric anomalies.

Figure 12 compares the degree 2 obtained from the observations of the local eigenfrequency of the spheroidal OS22 mode by Smith and Masters [1989] to 3SMAC prediction. The period of 0S22 is around $325 \mathrm{~s}$, a value much larger than that of the surface wave observations of $\mathrm{M} \& \mathrm{~T}$. This mode thus samples a relatively deep mantle at a depth where only few velocity anomalies (slabs and hotspots) are included in 3SMAC. The lithospheric anomalies are so strong, however, that they induce a $\pm 7 \mu \mathrm{Hz}$ amounting to half the observed signal. Although the predicted and observed signals are not in phase, 3SMAC reduces the variance of the observations of mode $0 \mathrm{~S} 22$ by $42 \%$.

Figure 13 summarizes some observations of the degree 2 from other spheroidal modes (0S22, 0S33, 0S43) [Smith and Masters, 1989]. Their observations and uncertainties are depicted by thick lines and 3SMAC predictions by open circles. The differences between the published values and the values plotted in this figure come from a different choice of spherical harmonics normalization. From Figure 13 it is clear that observations and 3SMAC predictions are different; it is satisfactory to see that anomalies other than those located in the lithosphere do exist. However, it is also clear that 3SMAC accounts for a very significant part of the signal, even though it has not been built from any seismic observations.

To check whether the degree 2 predicted by 3SMAC comes from the near-surface anomalies or from the slabs included in 3SMAC that cross in the transition zone, we separately computed the effects of the slabs and those of 3SMAC with slabs excluded. The a priori model without slabs accounts for only $25 \%$ of the observed variance for the $0 \mathrm{~S} 43$ mode ( $46 \%$ with slabs). The $C_{22}$ coefficient seems to be related mostly to the shallow lithospheric structure, whereas the other coefficients and specially the $S_{22}$ are the signatures of the presence of deep slabs. Increasing the anomaly associated with slabs leads to a better fit with eigenmode data. In fact, multiplying the slab contribution by a factor of 3.5 would explain $77 \%$ of the observations of mode 0S43. Even though the relationships used to convert slabs into seismic parameters have large uncertainties [Nataf and Ricard, 1996], these 

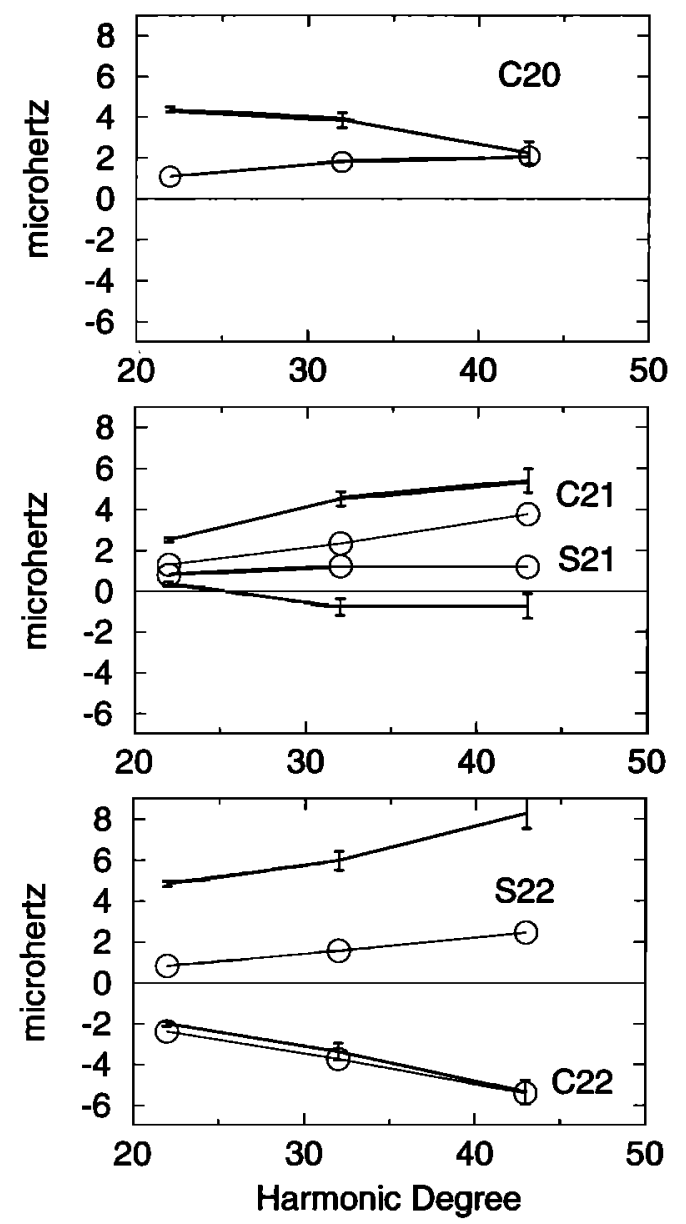

Figure 13. Degree 2 coefficients for various spheroidal modes observed by Smith and Masters [1989] or predicted from 3SMAC (circles). For all degrees and all periods, part of the observed signal is accounted for by 3SMAC.

uncertainties cannot account for such a factor of 3.5. Various possibilities can explain why the slabs might be associated with a 3 to 4 times larger anomaly. First, the slab model in 3SMAC has been built by using a very restrictive criterion. We include only slabs where seismicity is present, although slabs or portions of slabs without seismicity are clearly mapped by regional tomography [e.g., Spakman et al., 1988]. Second, slabs could be thickened in the transition zone, could be lying on the 670-km-depth interface [van der Hilst et al., 1991], or could be thickened below the transition zone as suggested by geoid modeling [Ricard et al., 1993].

Among the heterogeneities that have drawn the attention of geophysicists is the degree 6 of the upper mantle, which is a local maximum of the mantle heterogeneity spectra at long periods [Montagner and Romanowicz, 1993]. This degree is characteristic of the hotspot distribution [Richards et al., 1988]. The oceanic topography also exhibits a degree 6 in phase with the upper mantle tomography, the hotspot distribution, and the geoid [Cazenave and Thoraval, 1994; Montagner, 1994]. According to our results, the correlation between the distribution of our 97 hotspots and the velocity anomalies for Rayleigh waves at $200 \mathrm{~s}$ is 0.65 .
When the fact that 13 spectral coefficients are necessary to describe the degree 6 is taken into account, this correlation is significant with a confidence level of $99 \%$. The correlation of the synthetic map obtained from 3SMAC (Figure 5d) with hotspots is lower, being equal to 0.44 . However, this is still significant at $87 \%$. If we totally suppress the contribution of hotspots from 3SMAC and perform the same exercise of synthetic tomography, the correlation is only slightly affected and decreases further to 0.42 , which is significant at $85 \%$. Figure 14 depicts the degree 6 of the hostspot density in 3SMAC (Figure 14a), of the observed Rayleigh phase velocity at $200 \mathrm{~s}$ (Figure 14b), and of the prediction by 3SMAC of the same velocity when the hotspots have been excluded (Figure 14c). In fact, Figure 14c is very similar to the map obtained by including hotspots due to their weak contribution in 3SMAC (Gaussianshaped conduits with an excess temperature of $250 \mathrm{~K}$ over a diameter of $150 \mathrm{~km}$ ). The choice of a reduced list of hotspots does not change the correlations significantly. Using the 47 hotspots of Richards et al. [1988], we obtain for Rayleigh waves at $200 \mathrm{~s}$ a correlation of 0.71 between seismic observations and hotspots, of 0.57 between 3SMAC and hotspots, and of 0.55 between 3SMAC without hotspots and hotspots. These three correlations are significant at $99 \%, 96 \%$, and $95 \%$, respectively. These results suggest that the correlation between hotspots and upper mantle seismic velocity may not be indicative of a direct link but could be the indirect consequence of a series of correlations. For example, the hotspots may be triggered away from the past subductions, and the plume conduit may not cross the thick continental lithosphere. This behavior would imply a correlation of the observed hotspots with the ocean-continent distribution and thus indirectly with the upper mantle distribution. In this case, this correlation would not have a profound physical meaning.

\section{Conclusions}

In recent years, many tomographic models of the mantle structure have been published. The resolution of these models in the upper mantle has improved by a factor of 2 since the mid-eighties. The first generation of large-scale tomographic images was laterally expanded up to degree and order 6 or 8 and had a vertical resolution of about $200 \mathrm{~km}$ [e.g., Woodhouse and Dziewonski, 1984; Dziewonski, 1984; Nataf et al., 1984, 1986]. The latest results describe the upper mantle up to degree 20 (in fact, down to a correlation length of $2000 \mathrm{~km}$ ) [Montagner and Tanimoto, 1990], the whole mantle up to degree 12 [Su et al., 1992], or the upper mantle up to degree 36 (in fact, using a $5^{\circ}$ by $5^{\circ}$ grid) [Zhang and Tanimoto, 1993] or even better on a significant part of the mantle [Grand, 1994]. The vertical resolution has also increased accordingly.

These models have been used to infer the style of convection of the Earth. Some of the findings of seismologists that have important implications for geodynamics are the following:

1. The mantle heterogeneities are predominantly 


\section{HOTSPOT DENSITY (Degree 6)}

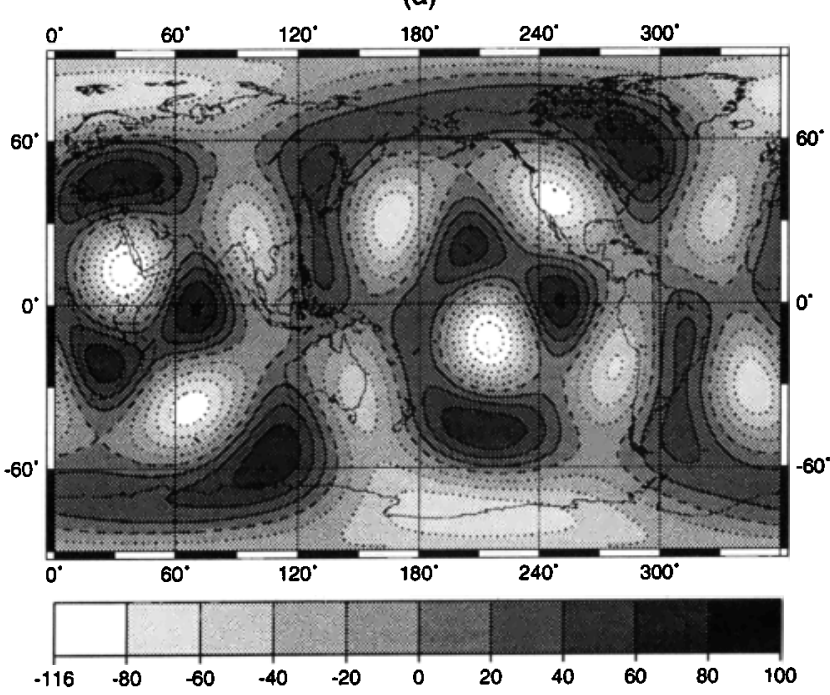

RAYLEIGH PH. VEL. at 200 s (Observed, Deg 6)

(b)

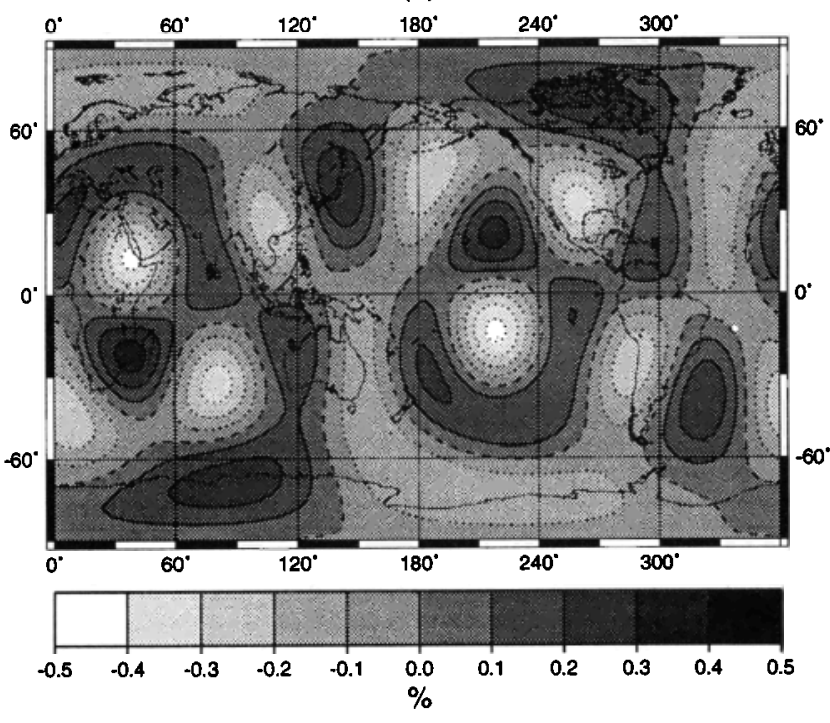

RAYLEIGH PH. VEL. at 200 s (3SMAC no Hotspots, Deg 6) (c)

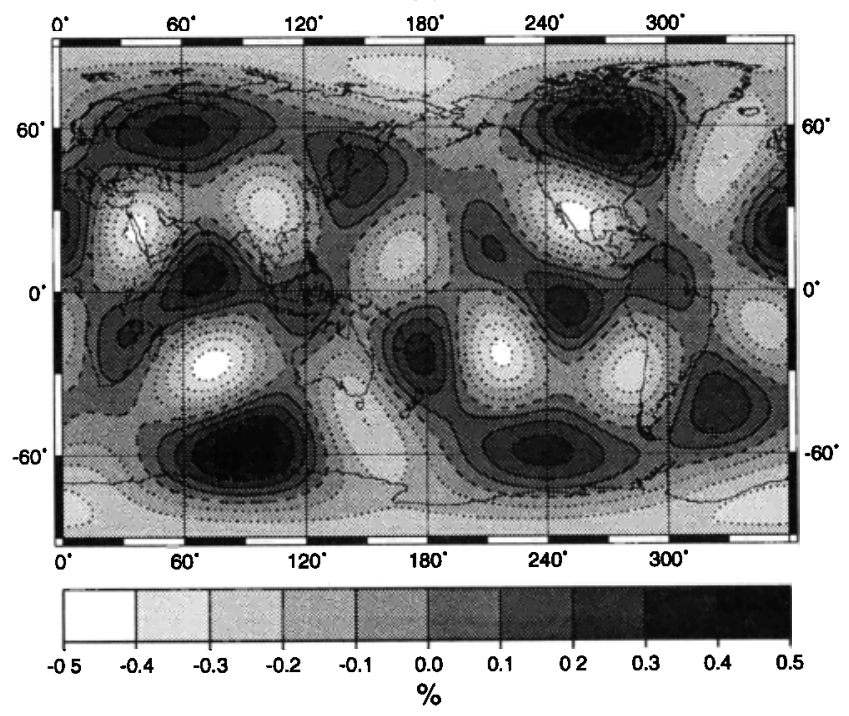

large-scale [Zhang and Tanimoto, 1991; Su and Dzzewon$s k i, 1991,1992]$.

2. A strong degree 2 anomaly lies in the transition zone [Masters et al., 1982].

3. A hot buoyant mantle extends below some fast ridges down to at least $300 \mathrm{~km}$ [Montagner and Tanimoto, 1991] and even to the deep lower mantle [Su et al., 1992].

4. The continental tectosphere has a thickness of more than $300 \mathrm{~km}$ [Jordan, 1975 ].

5. The hotspots have a strong degree 6 component in the transition zone [Montagner and Romanowncz, 1993].

These results and others may or may not agree with the ideas or preconceptions that geodynamicists have on mantle convection, but it is fair to say that they have significantly influenced our views on mantle behavior.

The approach followed in this paper and in the paper of Nataf and Ricard [1996] has been to try to specify the seismological implications of a standard geodynamical model rather than to directly interpret the seismological results. Therefore our conclusions underline the seismic observations that cannot be understood by using our basic knowledge of the lithospheric structure. Of course, our expertise on the subject is only based on the comparisons we performed between 3SMAC predictions and a given set of seismic observations, namely, $P$ and $\mathrm{S}$ travel time anomalies [Nataf and Ricard, 1996], Love and Rayleigh wave phase velocities, plus some lowdegree spheroidal eigenmodes. Our results regarding the five previously quoted findings do not entirely agree with them and in some cases do not agree with them at all.

1. As has already been suggested by various authors [Mochızuki, 1993; Snıeder, 1993; Nolet et al., 1994], the spectrum of heterogeneity deduced from surface wave tomography does not seem to be reliable for degrees larger than 10. A very strong smoothing is induced by the inversion. A better determination of small-scale structures can be reached by using shorter paths but is only slightly dependent on the densification of the long arc path coverage. Matching amplitudes and/or polarization [Laske et al., 1994] should also improve the inversion. Using a few thousand long path data can only give us an estimate of the first 6-8 degrees of the mantle heterogeneity. In our experiments with synthetics, even the structure at degree 3 is recovered at only $50 \%$.

2. The heterogeneities in the lithosphere are so strong that they drastically affect low-degree modes that should be sensitive to deeper structures. The observed degree 2 anomaly which is assumed to arise from anomalies in the transition zone has its minima (fast velocity) roughly located under the present subduction zones, in the West Pacific and under South America. The 3SMAC model does not predict the whole ampli-

Figure 14. Degree 6 comparison of (a) hotspot density distribution, (b) observed Rayleigh phase velocity at $200 \mathrm{~s}$, and (c) 3SMAC predicted Rayleigh phase velocity at $200 \mathrm{~s}$. All hotspot contributions were a priori excluded from 3SMAC; however, the correlation with the hotspot distribution (Figure 14a) is obvious. 
tude of the observations. However, some $50 \%$ of it can be ascribed to the near-surface structures: the very low velocities associated with the thick crust of the Tibetan Plateau and with the thin lithosphere under the PacificNazca-Antartica triple junction. This conclusion of a shallow origin of the $C_{22}$ term and a deeper origin of the $S_{22}$ term agrees with the findings of Romanowicz et al. [1987] and Romanowicz [1990], although it suggests an even shallower origin of $C_{22}$. It would be interesting to see whether slabs revealed by local tomography where no earthquakes are observed [e.g., van der Hilst et al., 1991] could explain the $S_{22}$ component.

3. Although other velocity anomalies are certainly located under the oceans, the age dependence of these anomalies is rather well predicted by a plate-cooling model. This conclusion is based on both PP-P and SS-S travel time anomalies [Nataf and Ricard, 1996] and on phase velocities of fundamental mode surface waves. The amplitudes of the anomalies under most of the ridges can be predicted from the temperature and pressure dependence of the velocity parameters (velocities, density, and attenuation) without explicitly requiring either partial melting or deep roots.

4. Under cratons, 3SMAC assumes a maximum depth of $300 \mathrm{~km}$ for a thermal lithosphere that has been cooling for $2 \mathrm{Ga}$. The phase velocity maps based on these assumptions predict roots that are too fast. This does not mean that the anomalies cannot extend deeper. We could have assumed deeper roots with shorter cooling times or invoked more complex relationships between geodynamic and seismological parameters. However, our results show that a lithospheric root less than 300 $\mathrm{km}$ is likely and certainly cannot be ruled out from the data we consider.

5. A satisfactory degree 6 is predicted by $3 S M A C$ which correlates with the hotspot distribution, although with a somewhat lower agreement than that of real observations. The degree 6 of 3SMAC can be predicted even if the contribution of the 97 hotspots of 3SMAC is removed. This finding suggests that most of the correlation between hotspots and long-period surface wave tomography might be a coincidence.

The fact that many of the anomalies detected from surface wave analysis can be explained by the basic ingredients of plate tectonics (3SMAC) is not surprising or disappointing. The main interest of tomography is not to tell us what we already know, the cooling of the oceanic floor or the difference between oceanic and continental lithosphere, but to localize the areas where the mantle structure is different from what could be expected from surface observations. Different examples can be taken from our tests. The Red Sea and Southeast China are clearly much slower than what is predicted by 3SMAC for all studied periods and for both Love and Rayleigh waves. Under continents the prediction of the lithospheric structure from the surface geology is hazardous. Greenland appears much slower than the Canadian shield, though it is often classified within the same cratonic zone. The Atlantic opening may have more deeply affected the Greenland lithosphere than we assumed.
The 3SMAC model has not been built to fit seismological data. No attempt has yet been made to modify the regionalizations, the thicknesses of the layers, or the choice of the conversion factors in order to satisfy seismic observations. Such modifications will be made only if they are not detrimental to the geodynamical coherency of the model. All comments from the seismological community aimed at improving 3SMAC are welcomed. We hope that 3SMAC can, at the least, be used as an easy way to test different seismological procedures (tomographic inversions, ray tracing, and so on) on a model that includes some of the complexities of the real Earth. We also hope 3SMAC can be used as a starting model for future inversions.

Acknowledgments. This study benefits from suggestions and data from many people. We thank in particular D. Anderson, P. Lognonné, G. Masters, G. Nolet, and Y. Zhang. We welcome further input to improve this model. 3SMAC and accompanying software is available from anonymous ftp to geoscope.ipgp.jussieu.fr. This research was supported by the INSU-CNRS program "Tomographie."

\section{References}

Anderson, D. L., Recent evidence concerning the structure and composition of the Earth's mantle, in Physics and Chemistry of the Earth, vol. 6., pp. 1-131, Pergamon, Tarrytown, N. Y., 1966.

Cazenave, C., and C. Thoraval, Mantle dynamics constrained by degree 6 surface topography, seismic tomography and geoid: inference on the origin of the South Pacific superswell, Earth Planet. Sci. Lett., 122, 207-220, 1994.

Clayton, R. W., and R. P. Comer, A tomographic analysis of mantle heterogeneities from body wave travel times (abstract), Eos. Trans. AGU, 64, 776, 1983.

Dziewonski, A. M., Mapping the lower mantle: Determination of lateral heterogeneity in $\mathrm{P}$ velocity up to degree and order 6, J. Geophys. Res., 89, 5929-5952, 1984.

Dziewonski, A. M., and D. L. Anderson, Preliminary reference Earth model, Phys. Earth Planet. Inter., 25, 297356, 1981.

Grand, S., Mantle structure beneath the Americas and surrounding oceans, J. Geophys. Res., 99, 11,591-11,621, 1994.

Ho Liu, P., J.-P. Montagner, and H. Kanamori, Comparison of iterative back-projection inversion and generalized inversion without blocks: Case studies in attenuation tomography, Geophys. J. Int., 97, 19-29, 1989.

Jordan, T. H., The continental tectosphere, Rev. Geophys., 13, 1-12, 1975.

Jordan, T. H., A procedure for estimating lateral variations from low frequency eigenspectra data, Geophys. J. R. Astron. Soc., 52, 441-455, 1978.

Jordan, T.H., Global tectonic regionalizations for seismological data analysis, Bull. Seismol. Soc. Am., 71, 11311141, 1981.

Kawakatsu, H., Can 'pure path' models explain free oscillation data?, Geophys. Res. Lett., 10, 186-189, 1983.

Laske, G., G. Masters, and W. Zürn, Frequency-dependent polarization measurements of long-period surface waves and their implications for global phase velocity maps, Phys. Earth Planet. Inter., 84, 111-137, 1994.

Masters, G., T. H. Jordan, P. G. Silver, and F. Gilbert, Aspherical Earth structure from fundamental spheroidalmode data, Nature, 298, 609-613, 1982.

Mochizuki, E., Spherical harmonics analysis in terms of line integral, Phys. Earth Planet. Inter., 76, 97-101, 1993. 
Montagner, J.-P., Can seismology tell us anything about convection in the mantle?, Rev. Geophys., 32, 115-137, 1994

Montagner, J.-P., and N. Jobert, Vectorial tomography, II, Application to the Indian Ocean, Geophys. J. R. Astron. Soc., 94, 309-344, 1988.

Montagner, J. P., and B. Romanowicz, Degrees 2, 4 and 6 inferred from seismic tomography, Geophys. Res. Lett., 20, 631-634, 1993.

Montagner, J.-P., and T. Tanimoto, Global anisotropy in the upper mantle inferred from the regionalization of the phase velocities, J. Geophys. Res., 95, 4797-4819, 1990.

Montagner, J. P., and T. Tanimoto, Global upper mantle tomography of seismic velocities and anisotropies, J. Geophys. Res., 96, 20,337-20,351, 1991.

Nakanishi, I., and D. L. Anderson, Measurements of mantle wave velocities and inversion for lateral heterogeneities and anisotropy, 1: Analysis of great circle phase velocities, J. Geophys. Res., 88, 10,267-10,283, 1984a.

Nakanishi, I. and D. L. Anderson, Measurements of mantle wave velocities and inversion for lateral heterogeneities and anisotropy, 2: Analysis by a single station method, Geophys. J. R. Astron. Soc., 78, 573-617, 1984b.

Nataf, H.-C., and Y. Ricard, 3SMAC: An a priori tomographic model of the upper mantle based on geophysical modeling, Phys. Earth Planet. Inter., in press, 1996.

Nataf, H.-C., I. Nakanishi, and D. L. Anderson, Anisotropy and shear-velocity heterogeneities in the upper mantle, Geophys. Res. Lett., 11, 109-112, 1984.

Nataf, H.-C., I. Nakanishi, and D. L. Anderson, Measurements of mantle wave velocities and inversion for lateral heterogeneities and anisotropy, 3: Inversion, J. Geophys. Res., 91, 7261-7307, 1986.

Nolet, G., S. P. Grand, and B. L. N. Kennett, Seismic heterogeneity in the upper mantle, J. Geophys. Res., 99, 23,753-23,766, 1994.

Peterson, J., H., and C. R. Hutt, Test and calibration of the Digital Worldwide Standardized Seismograph, U.S. Geol. Surv. Open File Rep., 1982.

Pulliam, R. J., and L. R. Johnson, What pattern of heterogeneity in the Earth's mantle can be revealed by seismic travel time tomography?, Phys. Earth Planet. Inter., 73, 109-151, 1992.

Ricard, Y., M. A. Richards, C. Lithgow-Bertelloni, and Y. Lestunff, A geodynamic model of mantle density heterogeneity, J. Geophys. Res., 98, 21,895-21,909, 1993.

Richards, M. A., B. H. Hager, and N. H. Sleep, Dynamically supported highs over hotspots: Theory and observations, J. Geophys. Res., 93, 7690-7708, 1988.

Romanowicz, B., The upper mantle degree 2: Constraints and inferences on attenuation tomography from global mantle wave measurements, $J$. Geophys. Res., 95, 11,05111,071, 1990.

Romanowicz, B., M. Cara, J. F. Fels, and D. Rouland, GEOSCOPE: A French initiative on long-period threecomponent seismic networks, Eos Trans. AGU, 65, 753$756,1984$.

Romanowicz, B., G. Roult, and T. Kohl, The upper mantle degree two pattern: Constraints from Geoscope fundamental spheroidal mode eigenfrequency and attenuation measurements, Geophys. Res. Lett., 14, 1219-1222, 1987.

Sato, Y., and T. Santo, World wide distribution of the group velocity of Rayleigh wave as determined by dispersion data, Bull. Earthquake. Res. Inst. Univ. Tokyo, 47, 31-41, 1969.

Smith, M. F., and G. Masters, Aspherical structure constraints from free oscillation frequency and attenuation measurements, J. Geophys. Res., 94, 1953-1976, 1989.

Snieder, R., Global inversions using normal modes and longperiod surface waves, in Seismic Tomography: Theory and
Practice, edited by H. M. Iyer and K. Hirahara, pp. 23-63, Chapman and Hall, New-York, 1993.

Snieder, R., J. Beckers, and F. Neele, The effect of smallscale structure on normal mode frequencies and global inversions, J. Geophys. Res., 96, 501-515, 1991.

Spakman, W., M. J. R. Wortel, and N. J. Vlaar, The Hellenic subduction zone: A tomographic image and its dynamic implications, Geophys. Res. Lett., 15, 60-63, 1988.

Stacey, F. D., Physics of the Earth, (2nd ed.), 329 pp., John Wiley, New York, 1977.

Su, W.-J., and A. M. Dziewonski, Predominance of longwavelength heterogeneity in the mantle, Nature, 352, 121126, 1991.

Su., W.-J., and A. M. Dziewonski, On the scale of mantle heterogeneity, Phys. Earth Planet. Inter., 74, 29-54, 1992.

Su, W.-J., R. J. Woodward, and A. M. Dziewonski, Deep origin of mid-ocean-ridge seismic velocity anomalies, $\mathrm{Na}$ ture, 360, 149-152, 1992.

Su, W.-J., R. J. Woodward, and A. M. Dziewonski, Degree 12 model of shear velocity heterogeneity in the mantle, $J$. Geophys. Res., 99, 6945-6980, 1994.

Tapponnier, P., G. Peltzer, A. Y. Le Dain, R. Armijo, and P. R. Cobbold, Propagating extrusion tectonics in Asia: New insights from simple experiments with plasticine, Geology, 10, 611-616, 1982.

Tarantola, A., and B. Valette, Generalized nonlinear inverse problems solved using the least squares criterion, Rev. Geophys., 20, 219-232, 1982.

Trampert, J., Comment on "Comparison of iterative backprojection inversion and generalized inversion without blocks: Case studies in attenuation tomography" by $\mathrm{P}$. Ho-Liu, J.-P. Montagner, and H. Kanamori, Geophys. J. Int., 103, 755-756, 1990.

Trampert, J., and J. H. Woodhouse, Global phase velocity maps of Love and Rayleigh waves between 40 and 150 seconds, Geophys. J. Int., 122, 675-690, 1995.

van der Hilst, R., R. Engdahl, W. Spakman, and G. Nolet, Tomographic imaging of subducted lithosphere below northwest Pacific island arcs, Nature, 353, 37-42, 1991.

Woodhouse, J. H., and A. M. Dziewonski, Mapping the upper mantle: Three-dimensional modeling of Earth structure by inversion of seismic waveforms, J. Geophys. Res., 89, 5953-5986, 1984.

Woodward, R. L., and G. Masters, Global upper mantle structure from long-period differential travel times, $J$. Geophys. Res., 96, 6351-6377, 1991.

Woodhouse, J. H., and Y. K. Wong, Amplitude, phase and path anomalies of mantle waves, Geophys. J. R. Astron. Soc., 87, 753-773, 1986.

Zhang, Y.-S., and T. Tanimoto, Global Love wave phase velocity variation and its significance to plate tectonics, Phys. Earth Planet. Inter., 66, 160-202, 1991.

Zhang, Y.-S., and T. Tanimoto, High-resolution global upper mantle structure and plate tectonics, J. Geophys. Res., 95, 6829-6851, 1993.

J.-P. Montagner, Institut de Physique du Globe, 4 place Jussieu, 75252, Paris Cedex 05, France. (e-mail: montagner@ipgp.jussieu.fr)

H.-C. Nataf, Laboratoire de Géologie, Ecole Normale Supérieure de Paris, 24 rue Lhomond, 75231, Paris Cedex 05, France. (e-mail: nataf@geophy.ens.fr)

Y. Ricard, Laboratoire de Géologie, Ecole Normale Supérieure de Lyon, 46 Allée d'Italie, 69364, Lyon Cedex 07, France. (e-mail: ricard@geologie.ens-lyon.fr)

(Received May 22, 1995; revised November 20, 1995; accepted November 29, 1995.) 